\title{
CONVERGENCE OF FINITE DIFFERENCE SCHEMES FOR VISCOUS AND INVISCID CONSERVATION LAWS WITH ROUGH COEFFICIENTS
}

\author{
Kenneth Hvistendahl Karlsen ${ }^{1}$ and Nils Henrik Risebro ${ }^{2}$
}

\begin{abstract}
We consider the initial value problem for degenerate viscous and inviscid scalar conservation laws where the flux function depends on the spatial location through a "rough" coefficient function $k(x)$. We show that the Engquist-Osher (and hence all monotone) finite difference approximations converge to the unique entropy solution of the governing equation if, among other demands, $k^{\prime}$ is in $B V$, thereby providing alternative (new) existence proofs for entropy solutions of degenerate convectiondiffusion equations as well as new convergence results for their finite difference approximations. In the inviscid case, we also provide a rate of convergence. Our convergence proofs are based on deriving a series of a priori estimates and using a general $L^{p}$ compactness criterion.
\end{abstract}

Mathematics Subject Classification. 65M06, 35L65, 35L45, 35K65.

Received: September 6, 2000.

\section{INTRODUCTION}

The main subject of this paper is finite difference schemes for computing the entropy solution of scalar viscous and inviscid conservation laws where the transport term depends explicitly on the spatial location. Such equations are of the form

$$
u_{t}+\operatorname{div} f(k, u)=\Delta A(u), \quad u(x, 0)=u_{0}(x), \quad(x, t) \in \Pi_{T}=\mathbb{R}^{d} \times(0, T),
$$

where the flux function $f(k, u)=\left(f_{1}\left(k^{1}, u\right), \ldots, f_{d}\left(k^{d}, u\right)\right)$ depends on the spatial location through the coefficient $k=k(x)$,

$$
k(x)=\left(k^{1}(x), \ldots, k^{d}(x)\right) .
$$

For the initial value problem (1.1) to be well-posed, we must require that the nonlinear elliptic operator $u \mapsto \Delta A(u)$ satisfies the degenerate ellipticity condition

$$
A(\cdot) \text { nondecreasing with } A(0)=0 .
$$

\footnotetext{
Keywords and phrases. Conservation law, degenerate convection-diffusion equation, entropy solution, finite difference scheme, convergence, error estimate.

${ }^{1}$ Department of Mathematics, University of Bergen, Johs. Brunsgt. 12, 5008 Bergen, Norway. e-mail: kennethk@math.uib.no; URL: http://www.mi.uib.no/ kennethk/

2 Department of Mathematics, University of Oslo, P.O. Box 1053, Blindern, 0316 Oslo, Norway. e-mail: nilshr@math.uio.no; URL: http://www.math.uio.no/ nilshr/
} 
Note that (1.2) implies that many well known nonlinear partial differential equations are special cases of (1.1). In particular (1.2) includes as special cases the inviscid conservation law, the heat equation, one-point degenerate porous medium type equations [43], two-point degenerate oil reservoir flow equations [15], and strongly degenerate convection-diffusion equations of the type arising in the theory of sedimentation-consolidation processes [4].

We recall that if (1.1) is allowed to degenerate at certain points, that is, $A^{\prime}(s)=0$ for some values of $s$, solutions are not necessarily smooth (but typically continuous) and weak solutions must be sought. On the other hand, if $A^{\prime}(s)$ is zero on an interval $[\alpha, \beta]$, (weak) solutions may be discontinuous and they are not uniquely determined by their initial data. Consequently, an entropy condition must be imposed to single out the physically correct solution. Roughly speaking, we call a function $u \in L^{1} \cap L^{\infty}$ an entropy solution of (1.1) if

$$
\begin{cases}\text { (i) } & \partial_{t}|u-c|+\operatorname{div}[\operatorname{sign}(u-c)(f(k, u)-f(k, c))]-\Delta|A(u)-A(c)| \\ & +\operatorname{sign}(u-c) \operatorname{div} f(k, c) \leq 0 \text { in } \mathcal{D}^{\prime} \forall c \in \mathbb{R}, \\ \text { (ii) } \quad \nabla A(u) \text { belongs to } L^{2} .\end{cases}
$$

We refer to Section 2.1 for a more precise statement of the definition of an entropy solution as well as precise conditions on $u_{0}, f, k, A$ ensuring that this definition makes sense. Relevant mathematical (existence and uniqueness) theory for entropy solutions can be found in $[3,5,28,42]$.

For the hyperbolic equation, the convergence analysis of numerical schemes has very long traditions and goes back to the 1950s. Being extremely selective, we mention only a few references related to finite difference and finite volume approximations. The case of finite difference schemes have been treated by Oleĭnik [40], Harten et al. [24], Kuznetsov [36], Crandall and Majda [12], Sanders [44], Lucier [37], Osher and Tadmor [41], Cockburn and Gremaud [10], and many others. The study of finite volume methods is more recent and have been conducted by Champier et al. [7], Vila [48], Cockburn et al. [8,9], Kröner and Rokyta [32], Kröner et al. [31], Noelle [38], Eymard et al. [21], and Chainais-Hillairet [6], as well as many others. Among the cited papers, only $[6,7,21,40]$ treat equations where the nonlinearity $f$ depends on the spatial position $x$ (and time $t$ ).

Although there seems to be an increasing interest in the (analysis of) numerical approximation of entropy solutions of degenerate parabolic equations, the amount of literature on the subject is at the moment modest. The (very recent) literature include papers by Evje and Karlsen [18], Holden et al. [25], and Holden et al. [26] on operator splitting methods (see also the lecture notes by Espedal and Karlsen [15]); Evje and Karlsen [16, 17, 19,20] on upwind difference schemes; Kurganov and Tadmor [35] on central difference schemes; Bouchut et al. [2] on kinetic BGK schemes; Afif and Amaziane [1] and Ohlberger [39] on finite volume methods; and Cockburn and Shu [11] on the local discontinuous Galerkin method. Strictly speaking, the authors of $[1,11,35]$ do not analyze their numerical methods within an entropy solution framework.

It is somewhat surprising that there have been few attempts up to very recently (confer the list of references given above) to develop a systematic treatment of mixed hyperbolic-parabolic partial differential equations within a unified mathematical (entropy solution) framework. In fact, the construction and analysis of numerical methods for first order hyperbolic and second order parabolic equations are usually considered as separate subject areas. In this work we demonstrate that it is possible to give a coherent treatment of numerical methods for such large class of nonlinear partial differential equations. Our main long-term goal is indeed to develop a consistent (mathematical/numerical) framework which is the same whether we are working with the hyperbolic case $\left(A^{\prime} \equiv 0\right)$, the parabolic case $\left(A^{\prime}>0\right)$, or with the mixed hyperbolic-parabolic case $\left(A^{\prime} \geq 0\right)$. In the present paper (see also $[16,17,19,20]$ ), we are concerned with finite difference schemes and their convergence analysis. For related work on other numerical methods for strongly degenerate parabolic equations, see the list of references given above.

To illustrate the results of this paper, we now state them in the one dimensional case (i.e., $d=1)$. For the general results, we refer to Sections 3 and 4. As a model difference scheme for (1.1), we consider the generalized upwind (Engquist-Osher) scheme

$$
\frac{u_{i}^{n+1}-u_{i}^{n}}{\Delta t}+\frac{f^{\mathrm{EO}}\left(k_{i+1 / 2}, u_{i}^{n}, u_{i+1}^{n}\right)-f^{\mathrm{EO}}\left(k_{i-1 / 2}, u_{i-1}^{n}, u_{i}^{n}\right)}{h}=\frac{A\left(u_{i+1}^{n}\right)-2 A\left(u_{i}^{n}\right)+A\left(u_{i-1}^{n}\right)}{h^{2}},
$$


where the so-called Engquist-Osher numerical flux [14] takes the form

$$
f^{\mathrm{EO}}\left(k, u_{1}, u_{2}\right)=\int_{0}^{u_{1}}\left(f_{u}(k, s) \vee 0\right) \mathrm{d} s+\int_{0}^{u_{2}}\left(f_{u}(k, s) \wedge 0\right) \mathrm{d} s+f(k, 0) .
$$

Here and in the following, $a \vee b=\max \{a, b\}$ and $a \wedge b=\min \{a, b\}$. Note that $k$ and $u$ are discretized on grids that are staggered with respect to each other. Concretely, we set

$$
u_{\Delta t}(x, t)=\sum_{i, n} \chi_{\left[x_{i-1 / 2}, x_{i+1 / 2}\right] \times\left[t_{n}, t_{n+1}\right\rangle} u_{i}^{n}, \quad k_{\Delta t}=\sum_{i} \chi_{\left[x_{i}, x_{i+1}\right\rangle} k_{i+1 / 2},
$$

where $k_{i+1 / 2}=k\left(x_{i+1 / 2}\right)$ and $x_{i}=i h$.

We have chosen to analyse the above scheme because of its simplicity. One can, however, adopt the method of proof developed in this paper and obtain similar results for other schemes (e.g., all monotone schemes) as well as more general equations. For example, under the assumption that $k \equiv 1$, Evje and Karlsen [20] have studied high order difference schemes (based on the MUSCL idea) for degenerate parabolic equations with source terms. One can easily combine the ideas in the present paper with those in [20] and obtain high order difference schemes for degenerate parabolic equations with source terms and $k=k(x)$ non-constant. Moreover, one can easily treat the case where $k$ possesses also a temporal dependence, i.e., $k=k(x, t)$. Although we consider only explicit schemes in this paper, one can adopt the techniques used herein to analyse semi-implicit and implicit schemes (the details will be presented elsewhere).

Assuming that $u_{0}, k$ and $k^{\prime}$ are in $B V$, we are able to show that the approximate solutions $\left\{u_{\Delta t}\right\}_{\Delta t>0}$ generated by our scheme converge strongly in $L_{\text {loc }}^{1}$ as $\Delta t \downarrow 0$ to the unique entropy solution. Furthermore, in the hyperbolic case, we show that this convergence has a rate. More precisely, we prove

Theorem 1.1. Let $u_{\Delta t}$ denote the function generated by the Engquist-Osher scheme. We assume that the time step $\Delta t$ is related to the spatial step $h$ through an appropriate CFL condition. If $u_{0}, k$ and $k^{\prime}$ are in $B V \cap L^{1} \cap L^{\infty}$, then

$$
u=\lim _{\Delta t \downarrow 0} u_{\Delta t}
$$

is the unique entropy solution to (1.1). Furthermore, if $A^{\prime} \equiv 0$, then

$$
\left\|u(\cdot, t)-u_{\Delta t}(\cdot, t)\right\|_{L^{1}(\mathbb{R})}=\mathcal{O}(\sqrt{\Delta t}) .
$$

We remark that Theorem 1.1 provides an existence result for entropy solutions of strongly degenerate parabolic equations which complements those in $[5,50]$. We also remark that the question of a convergence rate for the difference approximations to degenerate parabolic equations will be addressed elsewhere.

We now relate our results to the ones obtained by Evje and Karlsen $[17,19]$, who analyse monotone difference approximations of (1.1) in the special case $k \equiv 1$. In this case, the authors gave a fairly complete analysis for the one-dimensional equation under certain smoothness assumptions on the initial function $u_{0}$, in which case it actually holds that $A(u)$ belongs to the Hölder space $C^{1,1 / 2}(\mathbb{R} \times[0, T])$, and not merely $L^{2}\left(0, T ; H^{1}(\mathbb{R})\right)$ as follows from our analysis. We mention also the work [16] which generalizes the analysis in $[17,19]$ to the more difficult case of doubly nonlinear degenerate parabolic equations. In the present paper, we dispense with most of the smoothness assumptions on $u_{0}$ used in [17,19]. Moreover, in the multidimensional case, the authors of [19] do not prove that the limit $u$ of their monotone difference approximations satisfies (ii) in (1.3), a result that can be easily established by adopting the techniques developed in the present paper.

We continue with a few words about the proof of Theorem 1.1. The proof of the first part of Theorem 1.1 is based on deriving uniform $L^{\infty}, L^{1}$, and $B V$ bounds on the approximate solution $u_{\Delta t}$. Equipped with the $B V$ bound, we use the difference scheme itself and Kružkov's interpolation lemma [33] to show that $u_{\Delta t}$ is uniformly $L^{1}$ continuous in time. Kolmogorov's compactness criterion then immediately gives $L_{\text {loc }}^{1}$ convergence 
(along a subsequence) of $\left\{u_{\Delta t}\right\}_{\Delta t>0}$ to a function $u \in L^{1} \cap L^{\infty}$. Uniqueness of the entropy solution [5, 28] (see also Th. 2.1 herein) will imply that the whole sequence $\left\{u_{\Delta t}\right\}_{\Delta t>0}$ converges and not just a subsequence.

To ensure that the limit $u$ is the (unique) entropy solution in the sense of (1.3), we first prove that the difference scheme satisfies a so-called discrete (or cell) entropy inequality and hence it follows, by arguments analogous to the ones used to prove the Lax-Wendroff theorem, that the entropy condition (i) in (1.3) holds true for the limit $u$. In passing, we mention that the $B V$ regularity and the cell entropy inequalities are used to derive the error estimate in the hyperbolic case. In doing so, we follow Kuznetsov [36] and Kružkov [34].

Finally, we show that the limit $u$ satisfies (ii) in (1.3). The arguments needed to prove (ii) are rather involved and based on deriving a space estimate that is resemblant of the so-called weak $B V$ estimates employed by Champier et al. [7] and Eymard et al. [21] to prove convergence of finite volume methods on unstructured grids for the hyperbolic equation, see also $[1,22]$ for the diffusion equation. Equipped with our weak $B V$ estimate and an appropriate time estimate, Kolmogorov's compactness criterion implies strong $L_{\text {loc }}^{2}$ convergence (along a subsequence) of $\left\{A\left(u_{\Delta t}\right)\right\}_{\Delta t>0}$ to $A(u)$ and $\nabla A(u) \in L^{2}$.

Throughout this paper the coefficient $k(x)$ is not allowed to be discontinuous. In the one-dimensional hyperbolic case $\left(A^{\prime} \equiv 0\right)$ with $k(x)$ depending discontinuously on $x$, the equation (1.1) is often written as the following $2 \times 2$ system:

$$
u_{t}+f(k, u)_{x}=0, \quad k_{t}=0 .
$$

If $\partial f / \partial u$ changes sign, then this system is non-strictly hyperbolic. This complicates the analysis, and in order to prove compactness of approximated solutions a singular transformation $\Psi(k, u)$ has been used by several authors $[23,29,30,45]$. In these works convergence of the Glimm scheme and of front tracking was established in the case where $k$ may be discontinuous. Under weaker conditions on $k, e . g ., k^{\prime} \in B V$, and for $f$ convex in $u$, convergence of the one-dimensional Godunov method for (1.4) (not for (1.1)) was shown by Isaacson and Temple in [27]. Recently, convergence of the one-dimensional Engquist-Osher method for (1.1) was shown by Towers $[46,47]$ in the case where $k$ is piecewise continuous. In this case, the Kružkov entropy condition (1.3) no longer applies, and in [30] a wave entropy condition analogous to the Oleinik entropy condition introduced in [40] was used to obtain uniqueness. We intend to study the degenerate parabolic case (1.1) with a discontinuous $k(x)$ in future work.

The rest of this paper is organized as follows: in the next section we introduce (precisely) the notion of an entropy solution, and state the theorem regarding uniqueness and the $L^{1}$ contraction property of the solution operator to (1.1). We then proceed to show convergence and convergence rates of difference schemes for the hyperbolic equation. In the last section we show convergence of difference schemes for the degenerate parabolic equation.

Throughout this paper we denote by $C$ a generic constant, not depending on our discretization parameter $\Delta t$. Note that the actual value of $C$ may change from one line to the next during a calculation.

\section{Preliminaries}

In this section we first give a precise definition of an entropy solution, and then present some technical tools that we shall use.

\subsection{Definition of the entropy solution}

Throughout this paper we let $f_{i}\left(k^{i}, u\right)$ be smooth functions $\mathbb{R} \times \mathbb{R} \rightarrow \mathbb{R}$, and set $f(k, u)=\left(f_{1}\left(k^{1}, u\right), \ldots, f_{d}\left(k^{d}, u\right)\right)$. We assume that $A: \mathbb{R} \rightarrow \mathbb{R}$ is a function that satisfies

$$
A \in \operatorname{Liploc}_{\text {loc }}(\mathbb{R}) \text { and } A(\cdot) \text { is nondecreasing with } A(0)=0 .
$$

Concerning the flux function $f: \mathbb{R}^{d} \times \mathbb{R} \rightarrow \mathbb{R}^{d}$, we assume that $f \in C^{3}\left(\mathbb{R}^{d} \times \mathbb{R} ; \mathbb{R}^{d}\right)$ and that

$$
f_{i}, \partial_{u} f_{i}, \partial_{k} f_{i}, \partial_{u k} f_{i}, \partial_{u k k} f_{i} \in \operatorname{Lip}(\mathbb{R} \times \mathbb{R} ; \mathbb{R}), \quad \text { for } i=1, \ldots, d .
$$


Furthermore, we assume that the relevant Lipschitz constants are bounded by

$$
\left|\partial_{u} f_{i}\right| \leq L_{u}, \quad\left|\partial_{k} f_{i}\right| \leq L_{k}, \quad\left|\partial_{u k} f_{i}\right| \leq L_{u k}, \quad \text { and so on }
$$

for all $i$ and for some constants $L_{u}, L_{k}, L_{u k}$. Without explicitly mentioning this any more, we will always assume in this paper that $f(k, 0)=0$ for all $k$.

Regarding the coefficient $k$ we assume that

$$
k^{i} \in C\left(\mathbb{R}^{d}\right) \cap B V\left(\mathbb{R}^{d}\right), \quad \partial_{x_{j}} k^{i} \in L^{\infty}\left(\mathbb{R}^{d}\right) \cap B V\left(\mathbb{R}^{d}\right) \text { for all } i \text { and } j .
$$

Under the above assumptions we shall study difference approximations to (1.1). Following [28] (see also Carrillo [5]), an entropy solution is defined as follows:

Definition 2.1. An entropy solution of (1.1) is a measurable function $u=u(x, t)$ satisfying:

D.1 $u \in L^{1}\left(\Pi_{T}\right) \cap L^{\infty}\left(\Pi_{T}\right) \cap C\left(0, T ; L^{1}\left(\mathbb{R}^{d}\right)\right)$.

D.2 For all $c \in \mathbb{R}$, and all non-negative test functions $\varphi$ in $C_{0}^{\infty}\left(\Pi_{T}\right)$ the following entropy inequality holds:

$$
\begin{gathered}
\iint_{\Pi_{T}}\left(|u-c| \varphi_{t}\right. \\
+\operatorname{sign}(u-c)(f(k(x), u)-f(k(x), c)) \cdot \nabla \varphi+|A(u)-A(c)| \Delta \varphi \\
-\operatorname{sign}(u-c) \operatorname{div} f(k(x), c) \varphi) \mathrm{d} t \mathrm{~d} x \geq 0 .
\end{gathered}
$$

D.3 $A(u) \in L^{2}\left(0, T ; H^{1}\left(\mathbb{R}^{d}\right)\right)$.

D.4 Essentially as $t \rightarrow 0+$,

$$
\int_{\mathbb{R}^{d}}\left|u(x, t)-u_{0}(x)\right| \mathrm{d} x \rightarrow 0 .
$$

Remark 2.1. (i) Observe that when $A^{\prime} \equiv 0,(2.4)$ reduces to the well known entropy inequality for scalar conservation laws introduced by Kružkov [34] and Vol'pert [49].

(ii) Condition (D.4), i.e., that the initial datum $u_{0}$ should be taken by continuity, motivates the requirement of continuity with respect to $t$ in condition (D.1).

The following theorem from [28] shows that the initial value problem (1.1) is well posed:

Theorem 2.1. Assume that (2.1, 2.2) and (2.3) hold. Let $v, u \in L^{\infty}\left(0, T ; B V\left(\mathbb{R}^{d}\right)\right)$ be entropy solutions of (1.1) with initial data $v_{0}, u_{0} \in L^{1}\left(\mathbb{R}^{d}\right) \cap L^{\infty}\left(\mathbb{R}^{d}\right) \cap B V\left(\mathbb{R}^{d}\right)$, respectively. Then for almost all $t \in(0, T)$,

$$
\|v(\cdot, t)-u(\cdot, t)\|_{L^{1}\left(\mathbb{R}^{d}\right)} \leq\left\|v_{0}-u_{0}\right\|_{L^{1}\left(\mathbb{R}^{d}\right)}
$$

In particular, there exists at most one entropy solution of the initial value problem (1.1).

Remark 2.2. At the expense of loosing (2.5), the assumption that $v, u \in L^{\infty}\left(0, T ; B V\left(\mathbb{R}^{d}\right)\right)$ can be removed and uniqueness still holds, see [28].

\subsection{Some mathematical tools}

In this section we present some mathematical tools that we shall use in the analysis.

Let $u: \mathbb{R}^{d} \times(0, T) \rightarrow \mathbb{R}$ be a function such that $u(\cdot, t) \in L^{1}\left(\mathbb{R}^{d}\right)$ for all $t \in(0, T)$. By a modulus of continuity, we mean a nondecreasing continuous function $\nu: \mathbb{R}_{+}^{0} \rightarrow \mathbb{R}_{+}^{0}$ such that $\nu(0)=0$. We say that $u$ has a spatial modulus of continuity if

$$
\sup _{|\varepsilon| \leq y} \int_{\mathbb{R}^{d}}|u(x+\varepsilon, t)-u(x, t)| \mathrm{d} x \leq \nu(y ; u),
$$


(where $\nu$ may depend on $t$ ). We also say that $u$ has a temporal modulus of continuity if there is a modulus of continuity $\omega(\cdot ; u)$ such that for each $\tau \in(0, T)$,

$$
\sup _{0 \leq \varepsilon \leq \tau} \int_{\mathbb{R}^{d}}|u(x, t+\varepsilon)-u(x, t)| \mathrm{d} x \leq \omega(\tau ; u), \quad \forall t \in(0, T-\tau) .
$$

Let $\theta(r)$ be a smooth non-negative function of a single variable $r$ such that

$$
\theta(r)=\theta(-r), \quad \theta(r)=0, \quad \text { for }|r| \geq 1, \quad \text { and } \quad \int_{\mathbb{R}} \theta(r) \mathrm{d} r=1 .
$$

Let $\delta_{\varepsilon}(x)=\left(1 / \varepsilon^{d}\right) \theta(|x| / \varepsilon)$, and, with a slight abuse of notation, $\delta_{\varepsilon}(t)=(1 / \varepsilon) \theta(t / \varepsilon)$. Now define a test function $\varphi(x, y, t, s)$ by

$$
\varphi(x, y, t, s)=\delta_{\varepsilon}(x-y) \delta_{\varepsilon}(t-s)
$$

For a function $u=u(x, t)$, set

$$
\begin{aligned}
\lambda(u, c)= & -\iint_{\Pi_{T}}\left(|u-c| \varphi_{t}+\operatorname{sign}(u-c)(f(k, u)-f(k, c)) \varphi_{x}-\operatorname{sign}(u-c) f(k, c)_{x} \varphi\right) \mathrm{d} t \mathrm{~d} x \\
& +\left.\int_{\mathbb{R}}|u-c| \varphi\right|_{t=0} ^{t=T} \mathrm{~d} x .
\end{aligned}
$$

For two functions $u$ and $v$ we define the functional $\Lambda_{\varepsilon}(u, v)$ as

$$
\Lambda_{\varepsilon}(u, v)=\iint_{\Pi_{T}} \lambda(u, v(y, s)) \mathrm{d} s \mathrm{~d} y
$$

where $u=u(x, t)$ and $v=v(y, s)$. In passing, we note that if $A^{\prime} \equiv 0$, and $u$ is an entropy solution of (1.1), then

$$
\Lambda_{\varepsilon}(u, v) \leq 0
$$

For two arbitrary functions $u$ and $v$ we have the following result:

Lemma 2.1 (Kuznetsov's lemma). Assume that $k=\left(k^{1}, \ldots, k^{d}\right)$ is in $C\left(\mathbb{R}^{d}\right)$ and $k^{\prime}$ is in $L^{\infty}\left(\mathbb{R}^{d}\right)$ and that both $k$ and $k_{x_{i}}$ have moduli of continuity for all $i=1, \ldots, d$. If $u$ and $v$ are in $L^{1}\left(\Pi_{T}\right)$ and have moduli of continuity in space and time, then

$$
\begin{aligned}
\|u(\cdot, T)-v(\cdot, T)\|_{L^{1}\left(\mathbb{R}^{d}\right)} \leq & \|u(\cdot, 0)-v(\cdot, 0)\|_{L^{1}\left(\mathbb{R}^{d}\right)}+\Lambda_{\varepsilon}(u, v)+\Lambda_{\varepsilon}(v, u) \\
& +\frac{1}{2}[\nu(u(\cdot, 0) ; \varepsilon)+\nu(v(\cdot, 0) ; \varepsilon)+\nu(u(\cdot, T) ; \varepsilon)+\nu(v(\cdot, T) ; \varepsilon)] \\
& +\frac{1}{2}[\omega(u(\cdot, T) ; \varepsilon)+\omega(v(\cdot, T) ; \varepsilon)+\omega(u(\cdot, 0) ; \varepsilon)+\omega(v(\cdot, 0) ; \varepsilon)] \\
& +\|\operatorname{div} k\|_{L^{\infty}} L_{u} T \sup _{0 \leq t \leq T}(\nu(u(\cdot, t) ; \varepsilon)+\omega(u(\cdot, t) ; \varepsilon)) \\
& \left.+C T\left(\|\operatorname{div} k\|_{L^{\infty}} \nu(k ; \varepsilon)+\max _{i} \nu\left(k_{x_{i}} ; \varepsilon\right)\right)\right)
\end{aligned}
$$

where $\omega(\cdot ; \cdot)$ denotes a temporal modulus of continuity, and $\nu(\cdot ; \cdot)$ denotes a spatial modulus. The constant $C$ depends on $f_{k}$ and $f_{k k}$. 
Proof. We shall prove this lemma for $d=1$, the general proof is completely analogous. Remember that $\varphi_{x}=-\varphi_{y}$ and $\varphi_{t}=-\varphi_{s}$. By adding $\Lambda_{\varepsilon}(u, v)$ and $\Lambda_{\varepsilon}(v, u)$ we find that

$$
\begin{aligned}
\Lambda_{\varepsilon}(u, v)+\Lambda_{\varepsilon}(v, u)= & -\iint_{\Pi_{T}} \iint_{\Pi_{T}} \operatorname{sign}(u-v)\left[(f(k(x), u)-f(k(x), v)) \varphi_{x}-f(k(x), v)_{x} \varphi\right. \\
& \left.-(f(k(y), u)-f(k(y), v)) \varphi_{x}+f(k(y), u)_{y} \varphi\right] \mathrm{d} t \mathrm{~d} x \mathrm{~d} s \mathrm{~d} y \\
& +\iint_{\Pi_{T}} \int_{\mathbb{R}} \varphi(x, y, T, s)|u(x, T)-v(y, s)| \mathrm{d} x \mathrm{~d} s \mathrm{~d} y \\
& +\iint_{\Pi_{T}} \int_{\mathbb{R}} \varphi(x, y, t, T)|u(x, t)-v(y, T)| \mathrm{d} y \mathrm{~d} t \mathrm{~d} x \\
& -\iint_{\Pi_{T}} \int_{\mathbb{R}} \varphi(x, y, 0, s)|u(x, 0)-v(y, s)| \mathrm{d} x \mathrm{~d} s \mathrm{~d} y \\
& -\iint_{\Pi_{T}} \int_{\mathbb{R}} \varphi(x, y, t, 0)|u(x, t)-v(y, 0)| \mathrm{d} y \mathrm{~d} t \mathrm{~d} x .
\end{aligned}
$$

Using standard arguments, the four last terms will give the $\|u-v\|_{L^{1}}$ terms and the terms starting with $\frac{1}{2}[\ldots$ in (2.11). Regarding the remaining term, we follow Kružkov [34]. Let

$$
m(x, y, w)=f_{k}(k(x), w) k^{\prime}(x)-f_{k}(k(y), w) k^{\prime}(y) .
$$

Then we rewrite the square brackets in (2.12) as

$$
\begin{aligned}
{\left[(f(k(x), u)-f(k(x), v)) \varphi_{x}-\right.} & \left.f(k(x), v)_{x} \varphi-(f(k(y), u)-f(k(y), v)) \varphi_{x}+f(k(y), u)_{y} \varphi\right] \\
= & {\left[(f(k(y), u)-f(k(x), u)) \varphi_{y}+f(k(y), u)_{y} \varphi\right] } \\
& +\left[(f(k(y), v)-f(k(x), v)) \varphi_{x}-f(k(x), v)_{x} \varphi\right] \\
= & f_{k}(k(y), u) k^{\prime}(y)\left[(y-x) \varphi_{y}+\varphi\right]+m\left(\xi_{1}, y, u\right)(x-y) \varphi_{y} \\
& +f_{k}(k(y), v) k^{\prime}(y)\left[(y-x) \varphi_{x}-\varphi\right] \\
& +m(y, x, v) \varphi+m\left(\xi_{2}, y, v\right)(y-x) \varphi_{x} \\
= & -f_{k}(k(y), u) k^{\prime}(y)[(y-x) \varphi]_{x}+f_{k}(k(y), v) k^{\prime}(y)[(y-x) \varphi]_{x} \\
& +\left(m\left(\xi_{1}, y, u\right)+m\left(\xi_{2}, y, v\right)\right) \varphi_{y}+m(y, x, v) \varphi \\
= & {\left[k^{\prime}(y)\left(f_{k}(k(y), v)-f_{k}(k(y), u)\right)\right][(x-y) \varphi]_{x} } \\
& +\left(m\left(\xi_{1}, y, u\right)+m\left(\xi_{2}, y, v\right)\right)(x-y) \varphi_{y}+m(y, x, v) \varphi
\end{aligned}
$$

where $\left|\xi_{i}-y\right| \leq|x-y|$ for $i=1,2$. Let now

$$
F(u, v)=\operatorname{sign}(u-v)\left[k^{\prime}(y)\left(f_{k}(k(y), v)-f_{k}(k(y), u)\right)\right]
$$

and note that $F$ is Lipschitz continuous, with Lipschitz constant given by $\left\|k^{\prime}\right\|_{L^{\infty}} L_{u}$, in both arguments. Next, we obviously have that

$$
\iint_{\Pi_{T}} \iint_{\Pi_{T}} F(u(y, s), v(y, s))[(x-y) \varphi]_{x} \mathrm{~d} t \mathrm{~d} x \mathrm{~d} s \mathrm{~d} y=0 .
$$


Thus, our troublesome term reads

$$
\begin{aligned}
& \iint_{\Pi_{T}} \int_{\Pi_{T}}[F(u(x, t), v(y, s))-F(u(y, s), v(y, s))][(x-y) \varphi]_{x} \mathrm{~d} t \mathrm{~d} x \mathrm{~d} s \mathrm{~d} y \\
& \quad+\iint_{\Pi_{T}} \iint_{\Pi_{T}}\left(\operatorname{sign}(u-v)\left(m\left(\xi_{1}, y, u\right)+m\left(\xi_{2}, y, v\right)\right)(x-y) \varphi_{y}+m(y, x, v) \varphi\right) \mathrm{d} t \mathrm{~d} x \mathrm{~d} s \mathrm{~d} y .
\end{aligned}
$$

Now

$$
|m(x, y, u)| \leq L_{k}\left|k^{\prime}(x)-k^{\prime}(y)\right|+\left\|k^{\prime}\right\|_{L^{\infty}} L_{u k}|k(x)-k(y)| .
$$

Hence (2.14) is bounded by integrals of the form

$$
\iint_{\Pi_{T}} \int_{\Pi_{T}} \int_{T}|l(\xi)-l(y)|\left|(x-y) \delta_{\varepsilon}^{\prime}(x-y)\right| \delta_{\varepsilon}(t-s) \mathrm{d} t \mathrm{~d} x \mathrm{~d} s \mathrm{~d} y
$$

and

$$
\iint_{\Pi_{T}} \iint_{\Pi_{T}}|l(\xi)-l(y)| \delta_{\varepsilon}(x-y) \delta_{\varepsilon}(t-s) \mathrm{d} t \mathrm{~d} x \mathrm{~d} s \mathrm{~d} y
$$

where $\xi=x, \xi_{1}$ or $\xi_{2}$, and $l=k$ or $k^{\prime}$. Since $|x-y| \leq \varepsilon$ we have that $|\xi-y| \leq \varepsilon$ in (2.15) and (2.16), so they are both easily seen to be bounded by $\nu(l ; \varepsilon)$. The rest of $(2.13)$ is bounded as follows

$$
\begin{aligned}
& \iint_{\Pi_{T}} \iint_{\Pi_{T}}|F(u(x, t), v(y, s))-F(u(y, s), v(y, s))|\left|((x-y) \varphi)_{x}\right| \mathrm{d} t \mathrm{~d} x \mathrm{~d} s \mathrm{~d} y \\
& \leq\left\|k^{\prime}\right\|_{L^{\infty}} L_{u} \iiint \int|u(x, t)-u(y, s)|\left|((x-y) \varphi)_{x}\right| \mathrm{d} t \mathrm{~d} x \mathrm{~d} s \mathrm{~d} y \\
& \leq \frac{\left\|k^{\prime}\right\|_{L^{\infty}} L_{u}}{\varepsilon}\left[\int_{0}^{T} \iint_{|x-y| \leq \varepsilon}|u(x, t)-u(y, t)| \mathrm{d} x \mathrm{~d} y \mathrm{~d} t\right. \\
& \left.+\iint_{|t-s| \leq \varepsilon} \int|u(y, t)-u(y, s)| \mathrm{d} y \mathrm{~d} t \mathrm{~d} s\right] \\
& \leq\left\|k^{\prime}\right\|_{L^{\infty}} L_{u} T \sup _{0 \leq t \leq T}(\nu(u(\cdot, t) ; \varepsilon)+\omega(u(\cdot, t) ; \varepsilon)) .
\end{aligned}
$$

This concludes the proof of the lemma.

We need the following general $L^{1}$ and $L^{2}$ compactness criteria.

Lemma 2.2 ( $L_{\text {loc }}^{1}$ compactness lemma). Let $\left\{z_{h}\right\}_{h>0}$ be a sequence of functions defined on $\mathbb{R}^{d} \times(0, T)$ which satisfies:

(1) There exists a constant $C_{1}>0$ which is independent of $h$ such that

$$
\left\|z_{h}(\cdot, t)\right\|_{L^{1}\left(\mathbb{R}^{d}\right)} \text { and }\left\|z_{h}(\cdot, t)\right\|_{L^{\infty}\left(\mathbb{R}^{d}\right)} \leq C_{1}, \quad \forall t \in(0, T) .
$$


(2) There exists a spatial modulus of continuity $\nu$ which is independent of $h$ such that

$$
\left\|z_{h}(\cdot+y, t)-z_{h}(\cdot, t)\right\|_{L^{1}\left(\mathbb{R}^{d}\right)} \leq \nu\left(|y| ; z_{h}\right) \text { as } y \rightarrow 0, \quad \forall t \in(0, T) .
$$

(3) There exists a temporal modulus of continuity $\omega$ which is independent of $h$ such that

$$
\left\|z_{h}(\cdot, t+\tau)-z_{h}(\cdot, t)\right\|_{L^{1}\left(\mathbb{R}^{d}\right)} \leq \omega\left(\tau ; z_{h}\right), \quad \forall t \in(0, T-\tau) \text { whenever } \tau \in(0, T) .
$$

Then $\left\{z_{h}\right\}_{h>0}$ is compact in the strong topology of $L_{\text {loc }}^{1}\left(\mathbb{R}^{d} \times(0, T)\right)$. Moreover, any limit point of $\left\{z_{h}\right\}_{h>0}$ belongs to $L^{1}\left(\mathbb{R}^{d} \times(0, T)\right) \cap L^{\infty}\left(\mathbb{R}^{d} \times(0, T)\right) \cap C\left(0, T ; L^{1}\left(\mathbb{R}^{d}\right)\right)$.

Lemma 2.3 ( $L_{\text {loc }}^{2}$ compactness lemma). Let $\left\{z_{h}\right\}_{h>0}$ be a sequence of functions defined on $\mathbb{R}^{d} \times(0, T)$ which satisfies:

(1) There exists a constant $C_{1}>0$ which may depend on $T$, but not $h$, such that

$$
\left\|z_{h}\right\|_{L^{2}\left(\mathbb{R}^{d} \times(0, T)\right)} \leq C_{1}
$$

(2) There exists a constant $C_{2}>0$ which may depend on $T$ but not $h$ such that

$$
\left\|z_{h}(\cdot+y, \cdot)-z_{h}(\cdot, \cdot)\right\|_{L^{2}\left(\mathbb{R}^{d} \times(0, T)\right)} \leq C_{2}(|y|+h) \text { for all } y \text { as } h \downarrow 0 \text {. }
$$

(3) There exists a constant $C_{3}>0$ which may depend on $T$ but not $h$ such that

$$
\left\|z_{h}(\cdot, \cdot+\tau)-z_{h}(\cdot, \cdot)\right\|_{L^{2}\left(\mathbb{R}^{d} \times(0, T-\tau)\right)} \leq C_{3} \sqrt{\tau+h} \text { for all } \tau>0 \text { as } h \downarrow 0 \text {. }
$$

Then $\left\{z_{h}\right\}_{h>0}$ is compact in the strong topology of $L_{\text {loc }}^{2}\left(\mathbb{R}^{d} \times(0, T)\right)$. Moreover, any limit point of $\left\{z_{h}\right\}_{h>0}$ belongs to $L^{2}\left(0, T ; H^{1}\left(\mathbb{R}^{d}\right)\right)$.

To prove that the difference approximations possess some $L^{1}$ time continuity, we shall use the following lemma due to Kružkov [33].

Lemma 2.4 (Kružkov's interpolation lemma [33]). Let $z(x, t)$ be a bounded measurable function defined on $\mathbb{R}^{d} \times(0, T)$. For $t \in(0, T)$ assume that $z$ possesses a spatial modulus of continuity

$$
\int_{\mathbb{R}^{d}}|z(x+\varepsilon, t)-z(x, t)| \mathrm{d} x \leq \nu(|\varepsilon| ; z)
$$

where $\nu$ does not depend on $t$. Suppose that for any $\phi \in C_{0}^{\infty}\left(\mathbb{R}^{d}\right)$ and any $t_{1}, t_{2} \in(0, T)$,

$$
\left|\int_{\mathbb{R}^{d}}\left(z\left(x, t_{2}\right)-z\left(x, t_{1}\right)\right) \phi(x) \mathrm{d} x\right| \leq \operatorname{Const}_{T} \cdot\left(\sum_{|\alpha| \leq m} c_{\alpha}\left\|D^{\alpha} \phi\right\|_{L^{\infty}\left(R^{d}\right)}\right) \cdot\left|t_{2}-t_{1}\right|,
$$

where $\alpha$ denotes a multi-index, and $c_{\alpha}$ are constants not depending on $\phi$ or $t$. Then for any $t_{1}, t_{2} \in(0, T)$ and all $\varepsilon>0$

$$
\int_{\mathbb{R}^{d}}\left|z\left(x, t_{2}\right)-z\left(x, t_{1}\right)\right| \mathrm{d} x \leq C \cdot\left(\left|t_{2}-t_{1}\right| \sum_{|\alpha| \leq m} \frac{c_{\alpha}}{\varepsilon^{|\alpha|}}+\nu(z ; \varepsilon)\right)
$$

Proof. Let $\delta_{\varepsilon}(x)$ denote the usual mollifying kernel of radius $\varepsilon$, and let $\mathrm{d}(x)=z\left(x, t_{2}\right)-z\left(x, t_{1}\right)$. For $r>\varepsilon$, set

$$
\beta(x)= \begin{cases}\operatorname{sign}(\mathrm{d}(x)) & \text { for }|x| \leq r-\varepsilon \\ 0 & \text { otherwise }\end{cases}
$$


and set $\beta_{\varepsilon}=\beta * \delta_{\varepsilon}$. Then $\beta_{\varepsilon}$ is in $C_{0}^{\infty}\left(\mathbb{R}^{n}\right)$, has support inside the ball $B_{r}$, and we have the bound $\left|D^{\alpha} \beta_{\varepsilon}\right| \leq$ Const $/ \varepsilon^{|\alpha|}$. Also

$$
\begin{aligned}
\left|\int_{B_{r}} \mathrm{~d}(x) \mathrm{d} x\right| & \leq \int_{B_{r}}\left|\mathrm{~d}(x)-\beta_{\varepsilon}(x) \mathrm{d}(x)\right| \mathrm{d} x+\left|\int_{B_{r}} \beta_{\varepsilon}(x) \mathrm{d}(x) \mathrm{d} x\right| \\
& \leq \iint_{\mathbb{R}^{d}}|\mathrm{~d}(x)-\mathrm{d}(x-y)| \delta_{\varepsilon}(y) \mathrm{d} x \mathrm{~d} y+C\left|t_{2}-t_{1}\right| \sum_{|\alpha| \leq m} \frac{c_{\alpha}}{\varepsilon^{|\alpha|}} \\
& \leq C\left(\nu(\varepsilon ; z)+\left|t_{2}-t_{1}\right| \sum_{|\alpha| \leq m} \frac{c_{\alpha}}{\varepsilon^{|\alpha|}}\right) .
\end{aligned}
$$

Letting $r \uparrow \infty$, we obtain (2.19).

We shall also need the technical result:

Lemma 2.5 (Crandall and Tartar [13]). Let $(\Omega, \mu)$ be some measure space and let $D$ be a subset of $L^{1}(\Omega)$. Assume that if $u$ and $v$ are in $D$, then also $u \vee v$ is in $D$. Let $T$ be a map $D \rightarrow D$ such that

$$
\int_{\Omega} T(u) \mathrm{d} \mu=\int_{\Omega} u \mathrm{~d} \mu, \quad \forall u \in D .
$$

Then the following statements, valid for all $u$ and $v$ in $D$, are equivalent:

(i) If $u \leq v$, then $T(u) \leq T(v)$.

(ii) $\int_{\Omega}(\bar{T}(u)-T(v)) \vee 0 \mathrm{~d} \mu \leq \int_{\Omega}(u-v) \vee 0 \mathrm{~d} \mu$.

(iii) $\int_{\Omega}|T(u)-T(v)| \mathrm{d} \mu \leq \int_{\Omega}|u-v| \mathrm{d} \mu$.

\section{Difference approximations: The hyperbolic equation}

In this section we analyze a difference approximation to the solution of the hyperbolic equation

$$
u_{t}+\operatorname{div} f(k, u)=0, \quad u(x, 0)=u_{0}(x), \quad(x, t) \in \Pi_{T},
$$

where $k$ and $f$ satisfy (2.2) and (2.3) respectively. For simplicity, we shall assume that $u_{0}$ has compact support, which implies that all subsequent sums over $I$ are finite. To obtain results in the general case, we can use the stability result in Theorem 2.1 (these standard details will not be written out). We have chosen to analyze the hyperbolic equation separately since the analysis parallels the general case but is simpler. In the next section, where we consider the general case, we shall use several of the estimates obtained in this section. Furthermore, we provide a convergence rate in the hyperbolic case.

As already mentioned in the introduction, we use the Engquist-Osher scheme to make the analysis more concrete, but our methods can easily be adapted to general monotone schemes. For a scalar flux function $f_{i}(k, u)$, the Engquist-Osher flux $f_{i}^{\mathrm{EO}}[14]$ can be written as

$$
f_{i}^{\mathrm{EO}}(k, u, v)=\frac{1}{2}\left(f_{i}(k, u)+f_{i}(k, v)-\int_{u}^{v}\left|\partial_{u} f_{i}(k, s)\right| \mathrm{d} s\right) .
$$

To define the scheme, let $I$ be a multi-index $I=\left(i_{1}, \ldots, i_{d}\right)$ and set $e_{i}$ to be a multi-index with zeros everywhere except for a 1 at the $i$ th place. Furthermore, we choose a time step $\Delta t$ such that $N \Delta t=T$ and a spatial 
discretization parameter $h>0$. Letting $\lambda=\Delta t / h$, the Engquist-Osher scheme reads $(d \geq 2)$

$$
\begin{aligned}
u_{I}^{n+1} & =u_{I}^{n}-\lambda \sum_{i=1}^{d}\left[\bar{f}_{I+e_{i} / 2}^{\mathrm{EO}}-\bar{f}_{I-e_{i} / 2}^{\mathrm{EO}}\right] \\
& =u_{I}^{n}-\lambda \sum_{i=1}^{d} \frac{1}{2^{d-1}} \sum_{\substack{j \neq i \\
J=I \pm e_{j} / 2}}\left[f_{i}^{\mathrm{EO}}\left(k_{J+e_{i} / 2}^{i}, u_{I}^{n}, u_{I+e_{i}}^{n}\right)-f_{i}^{\mathrm{EO}}\left(k_{J-e_{i} / 2}^{i}, u_{I-e_{i}}^{n}, u_{I}^{n}\right)\right],
\end{aligned}
$$

where

$$
\bar{f}_{I+e_{i} / 2}^{\mathrm{EO}}=\frac{1}{2^{d-1}} \sum_{\substack{j \neq i \\ J=I \pm e_{j} / 2}} f_{i}^{\mathrm{EO}}\left(k_{J+e_{i} / 2}^{i}, u_{I}^{n}, u_{I+e_{i}}^{n}\right)
$$

For $d=1$, the Engquist-Osher scheme reads

$$
u_{i}^{n+1}=u_{i}^{n}-\lambda\left[f^{\mathrm{EO}}\left(k_{i+1 / 2}, u_{i}^{n}, u_{i+1}^{n}\right)-f^{\mathrm{EO}}\left(k_{i-1 / 2}, u_{i-1}^{n}, u_{i}^{n}\right)\right] .
$$

The approximate solution $u_{\Delta t}$ is then defined as

$$
u_{\Delta t}(x, t)=u_{I}^{n}, \quad \text { for }(x, t) \in \chi_{I} \times\left[t_{n}, t_{n+1}\right)
$$

where $\chi_{I}$ denotes the set

$$
\chi_{I}=\left\{x \in \mathbb{R}^{d} \mid\left(x_{I-e_{i} / 2}\right)_{i} \leq x_{i}<\left(x_{I+e_{i} / 2}\right)_{i}, i=1, \ldots, d\right\}
$$

and $x_{I}=h I$. We initialize the scheme by setting

$$
u_{I}^{0}=\frac{1}{\left|\chi_{I}\right|} \int_{\chi_{I}} u_{0}(x) \mathrm{d} x
$$

Note that $f_{i}^{\mathrm{EO}}(k, u, v)$ is not continuously differentiable in the first variable but merely Lipschitz. Therefore we introduce the following auxiliary numerical flux

$$
f_{i}^{\mathrm{EO}, \varepsilon}(k, u, v)=\frac{1}{2}\left(f_{i}(k, u)+f_{i}(k, v)-\int_{u}^{v}\left|\partial_{u} f_{i}(k, s)\right|_{\varepsilon} \mathrm{d} s\right)
$$

where $|\cdot|_{\varepsilon}$ is a smooth approximation to the absolute value function $|\cdot|$ such that

$$
\left.|| a|-| a\right|_{\varepsilon} \mid \leq \varepsilon \quad \text { and } \quad|a|=|a|_{\varepsilon} \quad \text { for }|a|>\varepsilon
$$


Note in particular that

$$
\left|f_{i}^{\mathrm{EO}}(k, u, v)-f_{i}^{\mathrm{EO}, \varepsilon}(k, u, v)\right| \leq|u-v| \varepsilon, \quad \forall k, u, v .
$$

This scheme can be analyzed as follows. Set

$$
\widehat{\sum_{i}}=\frac{1}{2^{d-1}} \sum_{\substack{j \neq i \\ J=I \pm e_{j} / 2}} \text { and } \quad \psi_{i}(k, u, v)=\frac{\partial f_{i}^{\mathrm{EO}, \varepsilon}}{\partial k}(k, u, v)
$$

Note that

$$
\left|\psi_{i}(k, u, v)\right| \leq L_{k}+\frac{1}{2}|u-v| L_{u k} .
$$

Then we define $F_{I}$ as the right hand side in (3.3), i.e.,

$$
u_{I}^{n+1}=F_{I}\left(u^{n}\right)
$$

Assuming the CFL condition

$$
\lambda \sum_{i=1}^{d} \max _{k, u}\left|\partial_{u} f_{i}(k, u)\right| \leq 1
$$

it is easy to show that $\frac{\partial F_{I}}{\partial u_{J}^{n}} \geq 0$ for all $J$. In other words, the Engquist-Osher scheme is monotone. Let $U^{n}=\max _{I}\left|u_{I}^{n}\right|$, then

$$
\begin{aligned}
\left|u_{I}^{n+1}\right| & =\left|F_{I}\left(u^{n}\right)\right| \leq F_{I}\left(U^{n}\right) \\
& =U^{n}+\lambda \sum_{i=1}^{d} \widehat{\sum_{i}}\left[f_{i}\left(k_{J+e_{i} / 2}^{i}, U^{n}\right)-f_{i}\left(k_{J-e_{i} / 2}^{i}, U^{n}\right)\right] \\
& \leq U^{n}+L_{k} \Delta t \sum_{i=1}^{d} \widehat{\sum_{i}}\left|k_{J+e_{i} / 2}^{i}-k_{J-e_{i} / 2}^{i}\right| \frac{1}{h} \leq U^{n}+L_{k} \Delta t \sum_{i=1}^{d}\left\|k_{x_{i}}^{i}\right\|_{L^{\infty}\left(\mathbb{R}^{d}\right)} .
\end{aligned}
$$

From this it follows that

$$
\left\|u_{\Delta t}(\cdot, T)\right\|_{L^{\infty}\left(\mathbb{R}^{d}\right)} \leq\left\|u_{0}\right\|_{L^{\infty}\left(\mathbb{R}^{d}\right)}+T L_{k} d \max _{i}\left\|k_{x_{i}}^{i}\right\|_{L^{\infty}\left(\mathbb{R}^{d}\right)} .
$$

Next, by the Crandall and Tartar lemma (see Lem. 2.5) and the monotonicity of $F_{I}$,

$$
\sum_{I}\left|F_{I}\left(u^{n}\right)-F_{I}(0)\right| \leq \sum_{I}\left|u_{I}^{n}\right|
$$


Hence

$$
h^{d} \sum_{I}\left|u_{I}^{n+1}\right| \leq h^{d} \sum_{I}\left|u_{I}^{n}\right|+h^{d} \lambda \sum_{i=1}^{d} \widehat{\sum_{i}}\left|f^{i}\left(k_{J+e_{i} / 2}^{i}, 0\right)-f^{i}\left(k_{J-e_{i} / 2}^{i}, 0\right)\right| \leq h^{d} \sum_{I}\left|u_{I}^{n}\right| .
$$

This means that

$$
\left\|u_{\Delta t}(\cdot, T)\right\|_{L^{1}\left(\mathbb{R}^{d}\right)} \leq\left\|u_{0}\right\|_{L^{1}\left(\mathbb{R}^{d}\right)}
$$

For any quantity $X_{I}$ defined on our grid let $D_{\ell} X_{I}$ denote the upward difference

$$
D_{\ell} X_{I}=X_{I+e_{\ell}}-X_{I}
$$

To bound the total variation of $u_{\Delta t}$ we again use the Crandall-Tartar lemma, which in this case gives

$$
\sum_{I}\left|F_{I+e_{\ell}}\left(u_{\cdot+e_{\ell}}^{n}\right)-F_{I+e_{\ell}}\left(u^{n}\right)\right| \leq \sum_{I}\left|u_{I+e_{\ell}}^{n}-u_{I}^{n}\right|
$$

Note that we have

$$
u_{I+e_{\ell}}^{n+1}=F_{I+e_{\ell}}\left(u_{\cdot+e_{\ell}}^{n}\right)
$$

and

$$
\sum_{I}\left|D_{\ell} u_{I}^{n+1}\right| \leq \sum_{I}\left|D_{\ell} u_{I}^{n}\right|+\left|\sum_{I}\left[F_{I+e_{\ell}}\left(u^{n}\right)-F_{I}\left(u^{n}\right)\right]\right|
$$

Before we start to estimate the difference on the right-hand side of (3.12), note that

$$
\begin{aligned}
\left|\psi_{i}\left(k_{1}, u, v\right)-\psi_{i}\left(k_{2}, u, v\right)\right|= & \frac{1}{2} \mid\left(\partial_{k k} f_{i}(\eta, u)+\partial_{k k} f_{i}(\nu, v)\right)\left(k_{1}-k_{2}\right) \\
& -\int_{u}^{v}\left(\left[\operatorname{sign}_{\varepsilon}\left(\partial_{u} f_{i}\left(k_{1}, s\right)\right)-\operatorname{sign}_{\varepsilon}\left(\partial_{u} f_{i}\left(k_{2}, s\right)\right)\right] \partial_{u k} f_{i}\left(k_{1}, s\right)\right. \\
& \left.+\operatorname{sign}_{\varepsilon}\left(\partial_{u} f_{i}\left(k_{2}, s\right)\right) \partial_{u k k} f_{i}(\gamma, s)\right) \mathrm{d} s \mid \\
\leq & \max _{k, u}\left|\partial_{k k} f_{i}(k, u)\right|\left|k_{1}-k_{2}\right|+\max _{k, u}\left|\partial_{u k} f_{i}(k, u)\right||u-v| \\
& +\frac{1}{2} \max _{k, u}\left|\partial_{u k k} f_{i}(k, u)\right||u-v|\left|k_{1}-k_{2}\right|
\end{aligned}
$$

where $\operatorname{sign}_{\varepsilon}(\cdot)$ denotes the derivative of $|\cdot|_{\varepsilon}$. Furthermore, we have

$$
\begin{aligned}
& \left|\psi_{i}\left(k, u_{1}, v\right)-\psi_{i}\left(k, u_{2}, v\right)\right| \leq \max _{k, u}\left|\partial_{k u} f_{i}(k, u)\right|\left|u_{1}-u_{2}\right| \\
& \left|\psi_{i}\left(k, u, v_{1}\right)-\psi_{i}\left(k, u, v_{2}\right)\right| \leq \max _{k, u}\left|\partial_{k u} f_{i}(k, u)\right|\left|v_{1}-v_{2}\right|
\end{aligned}
$$


Using the above estimates on $\psi_{i}$ and (3.6), there exist numbers $\xi_{J+e_{\ell} / 2 \pm e_{i} / 2}$ between $k_{J \pm e_{i} / 2}^{i}$ and $k_{J+e_{\ell} \pm e_{i} / 2}^{i}$ such that

$$
\begin{aligned}
& F_{I+e_{\ell}}\left(u^{n}\right)-F_{I}\left(u^{n}\right)=-\lambda \sum_{i=1}^{d} \widehat{\sum_{i}}\left[\left(f_{i}^{\mathrm{EO}}\left(k_{J+e_{\ell}+e_{i} / 2}^{i}, u_{I}^{n}, u_{I+e_{i}}^{n}\right)-f_{i}^{\mathrm{EO}}\left(k_{J+e_{i} / 2}^{i}, u_{I}^{n}, u_{I+e_{i}}^{n}\right)\right)\right. \\
& \left.-\left(f_{i}^{\mathrm{EO}}\left(k_{J+e_{\ell}-e_{i} / 2}^{i}, u_{I-e_{i}}^{n}, u_{I}^{n}\right)-f_{i}^{\mathrm{EO}}\left(k_{J-e_{i} / 2}^{i}, u_{I-e_{i}}^{n}, u_{I}^{n}\right)\right)\right] \\
& =\mathcal{O}(\varepsilon)-\lambda \sum_{i=1}^{d} \widehat{\sum_{i}}\left[\psi_{i}\left(\xi_{J+e_{\ell} / 2+e_{i} / 2}, u_{I}^{n}, u_{I+e_{i}}^{n}\right) D_{\ell} k_{J+e_{i} / 2}^{i}\right. \\
& \left.-\psi_{i}\left(\xi_{J+e_{\ell} / 2-e_{i} / 2}, u_{I-e_{i}}^{n}, u_{I}^{n}\right) D_{\ell} k_{J-e_{i} / 2}^{i}\right] \\
& =\mathcal{O}(\varepsilon)-\lambda \sum_{i=1}^{d} \widehat{\sum_{i}}\left[\psi_{i}\left(\xi_{J+e_{\ell} / 2+e_{i} / 2}, u_{I}^{n}, u_{I+e_{i}}^{n}\right) D_{i} D_{\ell} k_{J+e_{i} / 2}^{i}\right. \\
& \left.-D_{i} \psi_{i}\left(\xi_{J+e_{\ell} / 2-e_{i} / 2}, u_{I-e_{i}}^{n}, u_{I}^{n}\right) D_{\ell} k_{J-e_{i} / 2}^{i}\right] \\
& =\mathcal{O}(\varepsilon)-\lambda \sum_{i=1}^{d} \widehat{\sum_{i}}\left[\psi_{i}\left(\xi_{J+e_{\ell} / 2+e_{i} / 2}, u_{I}^{n}, u_{I+e_{i}}^{n}\right) D_{i} D_{\ell} k_{J+e_{i} / 2}^{i}\right. \\
& -\left\{\psi_{i}\left(\xi_{J+e_{\ell} / 2+e_{i} / 2}, u_{I}^{n}, u_{I+e_{i}}^{n}\right)-\psi_{i}\left(\xi_{J+e_{\ell} / 2-e_{i} / 2}, u_{I}^{n}, u_{I+e_{i}}^{n}\right)\right. \\
& +\psi_{i}\left(\xi_{J+e_{\ell} / 2-e_{i} / 2}, u_{I}^{n}, u_{I+e_{i}}^{n}\right)-\psi_{i}\left(\xi_{J+e_{\ell} / 2-e_{i} / 2}, u_{I}^{n}, u_{I}^{n}\right) \\
& \left.\left.+\psi_{i}\left(\xi_{J+e_{\ell} / 2-e_{i} / 2}, u_{I}^{n}, u_{I}^{n}\right)-\psi_{i}\left(\xi_{J+e_{\ell} / 2-e_{i} / 2}, u_{I-e_{i}}^{n}, u_{I}^{n}\right)\right\} D_{\ell} k_{J-e_{i} / 2}^{i}\right] \\
& \leq \mathcal{O}(\varepsilon)+\lambda \sum_{i=1}^{d} \widehat{\sum_{i}}\left[\left|\psi_{i}\left(\xi_{J+e_{\ell} / 2+e_{i} / 2}, u_{I}^{n}, u_{I+e_{i}}^{n}\right) D_{i} D_{\ell} k_{J+e_{i} / 2}^{i}\right|\right. \\
& -\left\{L_{k k}\left|D_{i} \xi_{J+e_{\ell} / 2-e_{i} / 2}\right|+L_{u k}\left|D_{i} u_{I}^{n}\right|+\frac{L_{u k k}}{2}\left|D_{i} u_{I}^{n} D_{i} \xi_{J+e_{\ell} / 2-e_{i} / 2}\right|\right. \\
& \left.\left.+L_{k u}\left(\left|D_{i} u_{I}^{n}\right|+\left|D_{i} u_{I-e_{i}}^{n}\right|\right)\right\}\left|D_{\ell} k_{J-e_{i} / 2}^{i}\right|\right] \text {. }
\end{aligned}
$$

As the above inequality holds for any $\varepsilon>0$, we can let $\varepsilon \downarrow 0$ and obtain

$$
\begin{aligned}
\left|F_{I+e_{\ell}}\left(u^{n}\right)-F_{I}\left(u^{n}\right)\right| \leq & \lambda \sum_{i=1}^{d} \widehat{\sum}_{i}\left[\left|\psi_{i}\left(\xi_{J+e_{\ell} / 2+e_{i} / 2}, u_{I}^{n}, u_{I+e_{i}}^{n}\right) D_{i} D_{\ell} k_{J+e_{i} / 2}^{i}\right|\right. \\
& -\left\{L_{k k}\left|D_{i} \xi_{J+e_{\ell} / 2-e_{i} / 2}\right|+L_{u k}\left|D_{i} u_{I}^{n}\right|+\frac{L_{u k k}}{2}\left|D_{i} u_{I}^{n} D_{i} \xi_{J+e_{\ell} / 2-e_{i} / 2}\right|\right. \\
& \left.\left.+L_{k u}\left(\left|D_{i} u_{I}^{n}\right|+\left|D_{i} u_{I-e_{i}}^{n}\right|\right)\right\}\left|D_{\ell} k_{J-e_{i} / 2}^{i}\right|\right] .
\end{aligned}
$$


Since we have that $\psi_{i}$ is bounded, we find that

$$
\begin{aligned}
\sum_{I}\left|D_{\ell} u_{I}^{n+1}\right| h^{d-1} \leq & \sum_{I}\left|D_{\ell} u_{I}^{n}\right| h^{d-1}+C \Delta t\left\{h^{d-2} \sum_{I} \sum_{i=1}^{d} \widehat{\sum_{i}}\left|D_{\ell} D_{i} k_{J+e_{i} / 2}^{i}\right|\right. \\
& \left.+h^{d-1} \max _{i, j}|| k_{x_{j}}^{i}\left\|_{L^{\infty}\left(\mathbb{R}^{d}\right)} \sum_{I} \sum_{i=1}^{d} \widehat{\sum_{i}}\left|D_{\ell} k_{J-e_{i} / 2}^{i}\right|+h^{d-1} \max _{i, j}\right\| k_{x_{j}}^{i} \|_{L^{\infty}\left(\mathbb{R}^{d}\right)} \sum_{I} \sum_{i=1}^{d}\left|D_{i} u_{I}^{n}\right|\right\}
\end{aligned}
$$

for some constant $C$ independent of $\Delta t$. The first and second sums inside $\{\cdots\}$ are bounded since we assume that $k^{i}$ and $k_{x_{j}}^{i}$ are in $B V\left(\mathbb{R}^{d}\right)$. By summing the above over $\ell=1, \ldots, d$, we find that

$$
\begin{aligned}
\left|u_{\Delta t}\left(\cdot, t_{n+1}\right)\right|_{B V\left(\mathbb{R}^{d}\right)}= & h^{d-1} \sum_{\ell=1}^{d} \sum_{I}\left|D_{\ell} u_{I}^{n+1}\right| \leq(1+C \Delta t)\left|u_{\Delta t}\left(\cdot, t_{n}\right)\right|_{B V\left(\mathbb{R}^{d}\right)} \\
& +C \Delta t\left(|k|_{B V\left(\mathbb{R}^{d}\right)}+\max _{i, j}\left|k_{x_{j}}^{i}\right|_{B V\left(\mathbb{R}^{d}\right)}\right) .
\end{aligned}
$$

Consequently,

$$
\left|u_{\Delta t}(\cdot, t)\right|_{B V\left(\mathbb{R}^{d}\right)} \leq C\left(\left|u_{0}\right|_{B V\left(\mathbb{R}^{d}\right)}+t\right), \quad \forall t \in(0, T)
$$

where $C$ does not depend on $\Delta t$.

Next, we shall use the scheme to show that $u_{\Delta t} \in C\left(0, T ; L^{1}\left(\mathbb{R}^{d}\right)\right)$ uniformly in $\Delta t$. This is done as follows

$$
\begin{aligned}
h^{d} \sum_{I}\left|u_{I}^{n+1}-u_{I}^{n}\right| \leq & \Delta t h^{d-1} \sum_{I} \widehat{\sum_{i}} \sum_{i=1}^{d}\left[\left|f_{i}^{\mathrm{EO}}\left(k_{J+e_{i} / 2}^{i}, u_{I}^{n}, u_{I+e_{i}}^{n}\right)-f_{i}^{\mathrm{EO}}\left(k_{J+e_{i} / 2}^{i}, u_{I-e_{i}}^{n}, u_{I}^{n}\right)\right|\right. \\
& \left.+\max \left|\frac{\partial f_{i}^{\mathrm{EO}}}{\partial k}\right|\left|D_{i} k_{J-e_{i} / 2}^{i}\right|\right] \\
\leq & C \Delta t\left(\left|u_{\Delta t}\right|_{B V\left(\mathbb{R}^{d}\right)}+|k|_{B V\left(\mathbb{R}^{d}\right)}\right)
\end{aligned}
$$

from which we obtain

$$
\left\|u_{\Delta t}(\cdot, t+\tau)-u_{\Delta t}(\cdot, t)\right\|_{L^{1}\left(\mathbb{R}^{d}\right)} \leq C \tau, \quad \forall t \in[0, T-\tau]
$$

By Lemma 2.2, we have that the sequence $\left\{u_{\Delta t}\right\}_{\Delta t>0}$ is compact in $L_{\mathrm{loc}}^{1}\left(\Pi_{T}\right)$. Moreover, any limit point of this sequence satisfies (D.1) and (D.4).

Next, we shall prove two cell entropy inequalities. The first one is based on Kružkov's entropies and will be used later to prove an error estimate. The second one is based on smooth $\left(C^{2}\right)$ convex entropies and will be used to show that the limit of any convergent subsequence of $\left\{u_{\Delta t}\right\}_{\Delta t>0}$ satisfies the entropy condition (D.2).

Let

$$
w_{I}^{n}=u_{I}^{n} \vee c, \quad v_{I}^{n}=u_{I}^{n} \wedge c, \quad w_{I}^{n+1}=F_{I}\left(w_{I}^{n}\right), \quad v_{I}^{n+1}=F_{I}\left(v_{I}^{n}\right), \quad c_{I}^{n+1}=F_{I}(c),
$$

where $F_{I}$ is defined in (3.7). Then we have that

$$
w_{I}^{n+1} \geq u_{I}^{n+1} \geq v_{I}^{n+1}, \quad v_{I}^{n+1} \leq c_{I}^{n+1} \leq w_{I}^{n+1} .
$$

This implies that

$$
\left|u_{I}^{n+1}-c_{I}^{n+1}\right| \leq w_{I}^{n+1}-v_{I}^{n+1}
$$


Now

$$
\begin{aligned}
& w_{I}^{n+1}-v_{I}^{n+1}=\left|u_{I}^{n}-c\right|-\lambda \sum_{i=1}^{d} \widehat{\sum_{i}}\left[\left(f_{i}^{\mathrm{EO}}\left(k_{J+e_{i} / 2}^{i}, u_{I}^{n} \vee c, u_{I+e_{i}}^{n} \vee c\right)\right.\right. \\
&\left.-f_{i}^{\mathrm{EO}}\left(k_{J+e_{i} / 2}^{i}, u_{I}^{n} \wedge c, u_{I+e_{i}}^{n} \wedge c\right)\right)-\left(f_{i}^{\mathrm{EO}}\left(k_{J-e_{i} / 2}^{i}, u_{I-e_{i}}^{n} \vee c, u_{I}^{n} \vee c\right)\right. \\
&\left.\left.\quad-f_{i}^{\mathrm{EO}}\left(k_{J-e_{i} / 2}^{i}, u_{I-e_{i}}^{n} \wedge c, u_{I}^{n} \wedge c\right)\right)\right] .
\end{aligned}
$$

Denoting the numerical entropy flux by

$$
q_{i}^{\mathrm{EO}}(k, u, v)=f_{i}^{\mathrm{EO}}(k, u \vee c, v \vee c)-f_{i}^{\mathrm{EO}}(k, u \wedge c, v \wedge c)
$$

and noting that

$$
\begin{aligned}
\left|u_{I}^{n+1}-c_{I}^{n+1}\right| & =\left|u_{I}^{n+1}-c+\lambda \sum_{i=1}^{d} \widehat{\sum_{i}}\left[f_{i}\left(k_{J+e_{i} / 2}^{i}, c\right)-f_{i}\left(k_{J-e_{i} / 2}^{i}, c\right)\right]\right| \\
& \geq \operatorname{sign}\left(u_{I}^{n+1}-c\right)\left(u_{I}^{n+1}-c+\lambda \sum_{i=1}^{d} \widehat{\sum_{i}}\left[f_{i}\left(k_{J+e_{i} / 2}^{i}, c\right)-f_{i}\left(k_{J-e_{i} / 2}^{i}, c\right)\right]\right) \\
& \geq\left|u_{I}^{n+1}-c\right|+\operatorname{sign}\left(u_{I}^{n+1}-c\right) \lambda \sum_{i=1}^{d} \widehat{\sum_{i}}\left[f_{i}\left(k_{J+e_{i} / 2}^{i}, c\right)-f_{i}\left(k_{J-e_{i} / 2}^{i}, c\right)\right],
\end{aligned}
$$

we find that

$$
\begin{aligned}
\left|u_{I}^{n+1}-c\right| \leq & \left|u_{I}^{n}-c\right|-\lambda \sum_{i=1}^{d} \widehat{\sum_{i}}\left[q_{i}^{\mathrm{EO}}\left(k_{J+e_{i} / 2}^{i}, u_{I}^{n}, u_{I+e_{i}}^{n}\right)-q_{i}^{\mathrm{EO}}\left(k_{J-e_{i} / 2}^{i}, u_{I-e_{i}}^{n}, u_{I}^{n}\right)\right] \\
& -\operatorname{sign}\left(u_{I}^{n+1}-c\right) \lambda \sum_{i=1}^{d} \widehat{\sum_{i}} D_{i} f_{i}\left(k_{J-e_{i} / 2}^{i}, c\right) .
\end{aligned}
$$

This discrete entropy inequality will be used later to derive an error estimate.

Let $U \in C^{2}$ be a convex entropy and let $\tilde{q}_{i}^{\mathrm{EO}}(k, u, v)$ denote the associated numerical entropy flux defined by

$$
\tilde{q}_{i}^{\mathrm{EO}}(k, u, v)=\int_{0}^{u} U^{\prime}(s)\left(\partial_{u} f_{i}(k, s) \vee 0\right) \mathrm{d} s+\int_{0}^{v} U^{\prime}(s)\left(\partial_{u} f_{i}(k, s) \wedge 0\right) \mathrm{d} s
$$

Let us write the scheme (3.3) as

$$
u_{I}^{n+1}=w_{I}^{n}-\lambda \sum_{i=1}^{d} \widehat{\sum_{i}}\left[f_{i}^{\mathrm{EO}}\left(k_{J+e_{i} / 2}^{i}, u_{I}^{n}, u_{I+e_{i}}^{n}\right)-f_{i}^{\mathrm{EO}}\left(k_{J-e_{i} / 2}^{i}, u_{I}^{n}, u_{I+e_{i}}^{n}\right)\right],
$$

where

$$
w_{I}^{n}=u_{I}^{n}-\lambda \sum_{i=1}^{d} \widehat{\sum_{i}}\left[f_{i}^{\mathrm{EO}}\left(k_{J-e_{i} / 2}^{i}, u_{I}^{n}, u_{I+e_{i}}^{n}\right)-f_{i}^{\mathrm{EO}}\left(k_{J-e_{i} / 2}^{i}, u_{I-e_{i}}^{n}, u_{I}^{n}\right)\right]
$$


From (3.18), it follows in a standard way using monotonicity (i.e., the CFL condition) that

$$
\frac{U\left(w_{I}^{n}\right)-U\left(u_{I}^{n}\right)}{\Delta t}+\sum_{i=1}^{d} \widehat{\sum_{i}} \frac{\tilde{q}_{i}^{\mathrm{EO}}\left(k_{J-e_{i} / 2}^{i}, u_{I}^{n}, u_{I+e_{i}}^{n}\right)-\tilde{q}_{i}^{\mathrm{EO}}\left(k_{J-e_{i} / 2}^{i}, u_{I-e_{i}}^{n}, u_{I}^{n}\right)}{h} \leq 0 .
$$

Using (3.17) and convexity of $U(\cdot)$, it follows that

$$
U\left(w_{I}^{n}\right) \geq U\left(u_{I}^{n+1}\right)+U^{\prime}\left(u_{I}^{n+1}\right) \lambda \sum_{i=1}^{d} \widehat{\sum_{i}}\left[f_{i}^{\mathrm{EO}}\left(k_{J+e_{i} / 2}^{i}, u_{I}^{n}, u_{I+e_{i}}^{n}\right)-f_{i}^{\mathrm{EO}}\left(k_{J-e_{i} / 2}^{i}, u_{I}^{n}, u_{I+e_{i}}^{n}\right)\right]
$$

Plugging this into (3.19), we get our desired cell entropy inequality

$$
\begin{aligned}
\frac{U\left(u_{I}^{n+1}\right)-U\left(u_{I}^{n}\right)}{\Delta t} & +\sum_{i=1}^{d} \widehat{\sum_{i}} \frac{\tilde{q}_{i}^{\mathrm{EO}}\left(k_{J+e_{i} / 2}^{i}, u_{I}^{n}, u_{I+e_{i}}^{n}\right)-\tilde{q}_{i}^{\mathrm{EO}}\left(k_{J-e_{i} / 2}^{i}, u_{I-e_{i}}^{n}, u_{I}^{n}\right)}{h} \\
& +U^{\prime}\left(u_{I}^{n+1}\right) \sum_{i=1}^{d} \widehat{\sum_{i}} \frac{f_{i}^{\mathrm{EO}}\left(k_{J+e_{i} / 2}^{i}, u_{I}^{n}, u_{I+e_{i}}^{n}\right)-f_{i}^{\mathrm{EO}}\left(k_{J-e_{i} / 2}^{i}, u_{I}^{n}, u_{I+e_{i}}^{n}\right)}{h} \\
& -\sum_{i=1}^{d} \widehat{\sum_{i}} \frac{\tilde{q}_{i}^{\mathrm{EO}}\left(k_{J+e_{i} / 2}^{i}, u_{I}^{n}, u_{I+e_{i}}^{n}\right)-\tilde{q}_{i}^{\mathrm{EO}}\left(k_{J-e_{i} / 2}^{i}, u_{I}^{n}, u_{I+e_{i}}^{n}\right)}{h} \leq 0 .
\end{aligned}
$$

We now multiply (3.20) by a nonnegative test function, do summation by parts, and then subsequently send $\Delta t \rightarrow 0$. We conclude that a limit point $u$ of $\left\{u_{\Delta t}\right\}_{\Delta t>0}$ satisfies the entropy condition

$$
\iint_{\Pi_{T}}\left(U(u) \varphi_{t}+q(k, u) \cdot \nabla \varphi-\left(U^{\prime}(u) f_{x}(k, u)-q_{x}(k, u)\right) \varphi\right) \mathrm{d} t \mathrm{~d} x \geq 0,
$$

where $q=\left(q_{1}, \ldots, q_{d}\right)$ satisfies

$$
\partial_{u} q(k, u)=U^{\prime}(u) \partial_{u} f(k, u) .
$$

Furthermore, $f_{x}, q_{x}$ denote the functions obtained by taking the divergence of $f(k(\cdot), u), q(k(\cdot), u)$, respectively. Since (3.21) holds for any convex $C^{2}$ entropy $U$ and corresponding entropy flux $q$, a standard approximation argument applied to $|\cdot-c|$ will reveal that $(2.4)$ (with $A^{\prime} \equiv 0$ ) holds for all $c \in \mathbb{R}$. Hence, by the uniqueness of the entropy solution, the whole sequence $\left\{u_{\Delta t}\right\}_{\Delta t>0}$ converges to the unique entropy solution.

Now we shall use the cell entropy inequality (3.15) and Kuznetsov's lemma (Lem. 2.1) to show that $u_{\Delta t}$ converges to the unique entropy solution at a rate of $\Delta t^{1 / 2}$. For simplicity we shall restrict our proof to the case of one space dimension, i.e., $d=1$. However, with some effort, the calculations given below can be generalized to the multi-dimensional case $d>1$.

Let $u(x, t)$ be the unique entropy solution to

$$
u_{t}+f(k, u)_{x}=0, \quad u(x, 0)=u_{0}(x)
$$

where $u_{0}$ and $k^{\prime}$ is of bounded variation, and let $u_{\Delta t}(x, t)$ and $k_{\Delta t}$ be as before. Now $\Lambda_{\varepsilon}\left(u, u_{\Delta t}\right) \leq 0$, and all the continuity moduli in Kuznetzov's lemma are linear in $\varepsilon$, therefore (2.11) reads

$$
\left\|u(\cdot, T)-u_{\Delta t}(\cdot, T)\right\|_{L^{1}(\mathbb{R})} \leq\left\|u_{0}-u_{\Delta t}(\cdot, 0)\right\|_{L^{1}(\mathbb{R})}+\Lambda_{\varepsilon}\left(u_{\Delta t}, u\right)+C \varepsilon
$$


where the constant $C$ does not depend on $\Delta t$. We must estimate $\Lambda_{\varepsilon}\left(u_{\Delta t}, u\right)$. Set

$$
\eta(u)=|u-c|, \quad q(k, u)=\operatorname{sign}(u-c)(f(k, u)-f(k, c)) .
$$

Multiplying the cell entropy inequality (3.15) by positive numbers $h \varphi_{i}^{n}$ with $\varphi_{i}^{n}=0$ for $|i|$ large, and summing over $i$ and $n=0, \ldots, N-1$ where $N \Delta t=T$, we find that

$$
\begin{aligned}
l\left(u_{\Delta t}, c\right):= & \sum_{i, n}\left[\left(\eta_{i}^{n+1}-\eta_{i}^{n}\right) \varphi_{i}^{n} h+\left(q^{\mathrm{EO}}\left(k_{i+1 / 2}, u_{i}^{n}, u_{i+1}^{n}\right)-q^{\mathrm{EO}}\left(k_{i-1 / 2}, u_{i-1}^{n}, u_{i}^{n}\right)\right) \varphi_{i}^{n} \Delta t\right. \\
& \left.+\operatorname{sign}\left(u_{i}^{n+1}-c\right)\left(f\left(k_{i+1 / 2}, c\right)-f\left(k_{i-1 / 2}, c\right)\right) \varphi_{i}^{n} \Delta t\right] \\
= & l_{1}+l_{2}+l_{3} \leq 0 .
\end{aligned}
$$

We also find that

$$
\begin{aligned}
\lambda_{\varepsilon}\left(u_{\Delta t}, c\right)= & \sum_{i, n}\left[\left(\eta_{i}^{n+1}-\eta_{i}^{n}\right) \int_{x_{i-1 / 2}}^{x_{i+1 / 2}} \varphi\left(x, t_{n}\right) \mathrm{d} x\right. \\
& +\left(q\left(k_{i+1 / 2}, u_{i+1}^{n}\right)-q\left(k_{i-1 / 2}, u_{i}^{n}\right)\right) \int_{t_{n}}^{t_{n+1}} \varphi\left(x_{i}, t\right) \mathrm{d} t \\
& +\left(q\left(k_{i+1 / 2}, u_{i+1}^{n}\right)-q\left(k_{i+1 / 2}, u_{i}^{n}\right)\right) \int_{t_{n}}^{t_{n+1}}\left(\varphi\left(x_{i+1 / 2}, t\right)-\varphi\left(x_{i}, t\right)\right) \mathrm{d} t \\
& \left.+\operatorname{sign}\left(u_{i}^{n+1}-c\right)\left(f\left(k_{i+1 / 2}, c\right)-f\left(k_{i-1 / 2}, c\right)\right) \int_{t_{n}}^{t_{n+1}} \varphi\left(x_{i}, t\right) \mathrm{d} t\right] \\
= & : \lambda_{1}+\lambda_{2,1}+\lambda_{2,2}+\lambda_{3} .
\end{aligned}
$$

Since $l\left(u_{\Delta t}, c\right) \leq 0$,

$$
\lambda_{\varepsilon}\left(u_{\Delta t}, u\right) \leq\left|\lambda_{\varepsilon}\left(u_{\Delta t}, u\right)-l\left(u_{\Delta t}, u\right)\right| \leq\left|\lambda_{1}-l_{1}\right|+\left|\lambda_{2,1}-l_{2}\right|+\left|\lambda_{3}-l_{3}\right|+\left|\lambda_{2,2}\right| .
$$

First we note that

$$
\left|\lambda_{2,2}\right| \leq L_{u} \sum_{i, n} \int_{x_{i}}^{x_{i+1 / 2}} \int_{t_{n}}^{t_{n+1}}\left|\delta_{\varepsilon}^{\prime}(x-y)\right| \delta_{\varepsilon}(t-s) \mathrm{d} t \mathrm{~d} x\left|u_{i+1}^{n}-u_{i}^{n}\right|
$$

and

$$
\iint_{\Pi_{T}}\left|\lambda_{2,2}\right| \mathrm{d} s \mathrm{~d} y \leq \frac{L_{u} T h}{\varepsilon}\left|u_{\Delta t}\right|_{B V(\mathbb{R})} .
$$

We choose the numbers $\varphi_{i}^{n}$ as

Using this we find that

$$
\varphi_{i}^{n}=\frac{1}{\Delta t h} \int_{x_{i-1 / 2}}^{x_{i+1 / 2}} \int_{t_{n}}^{t_{n+1}} \varphi(x, t) \mathrm{d} t \mathrm{~d} x
$$

$$
\begin{aligned}
\left|\lambda_{1}-l_{1}\right| & \leq \sum_{i, n}\left|\eta_{i}^{n+1}-\eta_{i}^{n}\right| \frac{1}{\Delta t} \int_{t_{n}}^{t_{n+1}} \int_{x_{i-1 / 2}}^{x_{i+1 / 2}}\left|\varphi(x, t)-\varphi\left(x, t_{n}\right)\right| \mathrm{d} x \mathrm{~d} t \\
& \leq \sum_{i, n}\left|\eta_{i}^{n+1}-\eta_{i}^{n}\right| \int_{t_{n}}^{t_{n+1}} \int_{x_{i-1 / 2}}^{x_{i+1 / 2}}\left|\varphi_{t}\right| \mathrm{d} x \mathrm{~d} t .
\end{aligned}
$$


Therefore,

$$
\begin{aligned}
\iint_{\Pi_{T}}\left|\lambda_{1}-l_{1}\right| \mathrm{d} s \mathrm{~d} y & \leq \sum_{i, n}\left|\eta_{i}^{n+1}-\eta_{i}^{n}\right| \int_{t_{n}}^{t_{n+1}} \int_{x_{i-1 / 2}}^{x_{i+1 / 2}} \iint\left|\delta_{\varepsilon}^{\prime}(t-s)\right| \delta_{\varepsilon}(x-y) \mathrm{d} s \mathrm{~d} y \mathrm{~d} x \mathrm{~d} t \\
& \leq \sum_{i, n}\left|\eta_{i}^{n+1}-\eta_{i}^{n}\right| C \frac{\Delta t h}{\varepsilon} \\
& \leq C \frac{\Delta t}{\varepsilon}
\end{aligned}
$$

We continue with

$$
\begin{aligned}
&\left|\lambda_{2,1}-l_{2}\right| \leq \sum_{i, n}\left[\int_{t_{n}}^{t_{n+1}} \varphi\left(x_{i}, t\right) \mathrm{d} t \times \mid\left(q\left(k_{i+1 / 2}, u_{i+1}^{n}\right)-q^{\mathrm{EO}}\left(k_{i+1 / 2}, u_{i}^{n}, u_{i+1}^{n}\right)\right)\right. \\
&-\left(q\left(k_{i-1 / 2}, u_{i}^{n}\right)-q^{\mathrm{EO}}\left(k_{i-1 / 2}, u_{i-1}^{n}, u_{i}^{n}\right)\right) \mid \\
&\left.+\left|\int_{t_{n}}^{t_{n+1}} \varphi\left(x_{i}, t\right) \mathrm{d} t-\varphi_{i}^{n} \Delta t\right|\left|q^{\mathrm{EO}}\left(k_{i+1 / 2}, u_{i}^{n}, u_{i+1}^{n}\right)-q^{\mathrm{EO}}\left(k_{i-1 / 2}, u_{i-1}^{n}, u_{i}^{n}\right)\right|\right] \\
&+\sum_{i, n}\left[\left|q\left(k_{i+1 / 2}, u_{i+1}^{n}\right)-q^{\mathrm{EO}}\left(k_{i+1 / 2}, u_{i}^{n}, u_{i+1}^{n}\right)\right| \int_{t_{n}}^{t_{n+1}} \int_{x_{i}}^{x_{i+1}}\left|\varphi_{x}\right| \mathrm{d} x \mathrm{~d} t\right. \\
&\left.+\int_{t_{n}}^{t_{n+1}} \int_{x_{i-1 / 2}}^{x_{i+1 / 2}}\left|\varphi_{x}\right| \mathrm{d} x \mathrm{~d} t\left|q^{\mathrm{EO}}\left(k_{i+1 / 2}, u_{i}^{n}, u_{i+1}^{n}\right)-q^{\mathrm{EO}}\left(k_{i-1 / 2}, u_{i-1}^{n}, u_{i}^{n}\right)\right|\right] \\
& \leq \sum_{i, n}\left[L_{u}^{t_{n+1}} \int_{t_{n}}^{x_{i+1}}\left|\varphi_{x_{i}}\right| \mathrm{d} x \mathrm{~d} t\left|u_{i+1}^{n}-u_{i}^{n}\right|\right. \\
&\left.+C \int_{t_{n}}^{t_{n+1}} \int_{x_{i-1 / 2}}^{x_{i+1 / 2}}\left|\varphi_{x}\right| \mathrm{d} x \mathrm{~d} t\left\{\left|k_{i+1 / 2}-k_{i-1 / 2}\right|+\left|u_{i}^{n}-u_{i-1}^{n}\right|+\left|u_{i+1}^{n}-u_{i}^{n}\right|\right\}\right]
\end{aligned}
$$

Consequently

$$
\iint_{\Pi_{T}}\left|\lambda_{2,1}-l_{2}\right| \mathrm{d} y \mathrm{~d} s \leq C\left(\left|u_{\Delta t}\right|_{B V(\mathbb{R})}+|k|_{B V(\mathbb{R})}\right) \frac{h}{\varepsilon} \leq C \frac{h}{\varepsilon} .
$$

Finally we estimate

$$
\begin{aligned}
\left|\lambda_{3}-l_{3}\right| \leq & \sum_{i, n}\left[\left|f\left(k_{i+1 / 2}, c\right)-f\left(k_{i-1 / 2}, c\right)\right| \frac{1}{h} \int_{t_{n}}^{t_{n+1}} \int_{x_{i-1 / 2}}^{x_{i+1 / 2}}\left|\varphi\left(x_{i}, t\right)-\varphi(x, t)+\varphi(x, t)-\varphi(x, t-\Delta t)\right| \mathrm{d} x \mathrm{~d} t\right. \\
& \left.+\sum_{i}\left|f\left(k_{i+1 / 2}, c\right)-f\left(k_{i-1 / 2}, c\right)\right|\left\{\int_{0}^{\Delta t} \varphi\left(x_{i}, t\right) \mathrm{d} t+\Delta t \varphi_{i}^{N-1}\right\}\right] \\
\leq & L_{k} \sum_{i, n}\left[\left|k_{i+1 / 2}-k_{i-1 / 2}\right| \int_{t_{n}}^{t_{n+1}} \int_{x_{i-1 / 2}}^{x_{i+1 / 2}} h\left|\varphi_{x}\right|+\Delta t\left|\varphi_{t}\right| \mathrm{d} x \mathrm{~d} t\right. \\
& \left.+L_{k} \sum_{i}\left|k_{i+1 / 2}-k_{i-1 / 2}\right|\left\{\int_{0}^{\Delta t} \varphi\left(x_{i}, t\right) \mathrm{d} t+\Delta t \varphi_{i}^{N-1}\right\}\right] .
\end{aligned}
$$


Therefore

$$
\begin{aligned}
\iint_{\Pi_{T}}\left|\lambda_{3}-l_{3}\right| \mathrm{d} s \mathrm{~d} y & \leq L_{k} \sum_{i, n}\left|k_{i+1 / 2}-k_{i-1 / 2}\right| \frac{h^{2} \Delta t+h \Delta t^{2}}{\varepsilon}+\sum_{i}\left|k_{i+1 / 2}-k_{i-1 / 2}\right| C \Delta t \\
& \leq C|k|_{B V(\mathbb{R})}\left(\frac{h^{2}+h \Delta t}{\varepsilon}+\Delta t\right) .
\end{aligned}
$$

Collecting the bounds (3.29-3.31), and (3.32), we find that

$$
\Lambda_{\varepsilon}\left(u_{\Delta t}, u\right) \leq C\left(\Delta t+\frac{h+\Delta t+h^{2}+h \Delta t}{\varepsilon}\right),
$$

for some constant $C$ not depending on $\Delta t$ or $h$. Since we assume that $u_{0}$ has bounded variation,

$$
\left\|u(\cdot, 0)-u_{\Delta t}(\cdot, 0)\right\|_{L^{1}(\mathbb{R})} \leq C h,
$$

and using this in (3.23) as well as $h=\Delta t / \lambda$ we arrive at the inequality

$$
\left\|u(\cdot, T)-u_{\Delta t}(\cdot, T)\right\|_{L^{1}(\mathbb{R})} \leq C\left(\varepsilon+\frac{\Delta t}{\varepsilon}\right),
$$

which is minimized by setting $\varepsilon=\sqrt{\Delta t}$.

The main result of this section is summed up in the following theorem, which is stated for multi-dimensional equations:

Theorem 3.1. Assume that $f$ and $k$ satisfy (2.2) and (2.3), respectively. Moreover, assume that $u_{0}$ is a function in $L^{1}\left(\mathbb{R}^{d}\right) \cap L^{\infty}\left(\mathbb{R}^{d}\right) \cap B V\left(\mathbb{R}^{d}\right)$. Let u be the unique entropy solution of (3.1). If the CFL condition (4.16) holds, then there exists a constant $C$, depending on $k, k_{x_{i}}, u_{0}, f$ and $T$, but not on $\Delta t$, such that

$$
\left\|u_{\Delta t}(\cdot, T)-u(\cdot, T)\right\|_{L^{1}\left(\mathbb{R}^{d}\right)} \leq C \sqrt{\Delta t},
$$

where the Engquist-Osher approximate solution $u_{\Delta t}$ is build from (3.3) and (3.5).

Remark 3.1. The assumptions on $k=\left(k^{1}, \ldots, k^{d}\right)$ in Theorem 3.1 are (slightly) less restrictive than those used in $[1,6,7,21]$ for finite volume methods.

\section{Difference approximations: The Degenerate Parabolic equation}

In this section we analyse the Engquist-Osher scheme for the degenerate parabolic equation (1.1). Again we shall assume that $u_{0}$ has compact support so that all subsequent sums over $I$ are finite. To obtain results for the general case, we can use the stability result in Theorem 2.1.

Let $\lambda=\Delta t / h$ (as usual) and $\mu=\Delta t / h^{2}$, then this the scheme reads $(d \geq 2)$

$$
\begin{aligned}
u_{I}^{n+1} & =u_{I}^{n}-\lambda \sum_{i=1}^{d} \widehat{\sum_{i}} D_{i} f_{i}^{\mathrm{EO}}\left(k_{J-e_{i} / 2}^{i}, u_{I-e_{i}}^{n}, u_{I}^{n}\right)+\mu \sum_{i=1}^{d} D_{i}^{2} A\left(u_{I-e_{i}}^{n}\right) \\
& =: G_{I}\left(u^{n}\right),
\end{aligned}
$$

where $D_{i}$ denotes the usual upwind difference operator, see (3.11). For $d=1$, the scheme reads

$$
u_{i}^{n+1}=u_{i}^{n}-\lambda D f^{\mathrm{EO}}\left(k_{i-1 / 2}, u_{i-1}^{n}, u_{i}^{n}\right)+\mu D^{2} A\left(u_{i-1}^{n}\right) .
$$


Let $u_{\Delta t}$ be the piecewise constant function defined by (4.1) and (3.5).

As a starting point we assume that the following CFL condition holds

$$
\mathrm{CFL}=\lambda \sum_{i=1}^{d} \max _{k, u}\left|\partial_{u} f_{i}(k, u)\right|+2 \mu d \max _{u} A^{\prime}(u) \leq 1
$$

Remark 4.1. The CFL condition (4.2) will be sufficient to establish the convergence of the sequence $\left\{u_{\Delta t}\right\}_{\Delta t>0}$ and moreover that a limit point $u$ of this sequence satisfies (D.1), (D.2), and (D.4). However, to prove that $u$ obeys (D.3), we shall later need a slightly stronger CFL condition (see (4.16) below).

Now it is easy to see that the CFL condition (4.2) implies that $\frac{\partial G_{I}}{\partial u_{J}^{n}} \geq 0$ for all $J$ and the scheme (4.1) is monotone. In the same manner as the bounds $(3.9,3.10)$, and $(3.13)$, we show that

$$
\left\|u_{\Delta t}(\cdot, t)\right\|_{L^{\infty}\left(\mathbb{R}^{d}\right)} \leq C, \quad\left\|u_{\Delta t}(\cdot, t)\right\|_{L^{1}\left(\mathbb{R}^{d}\right)} \leq C, \quad\left|u_{\Delta t}(\cdot, t)\right|_{B V\left(\mathbb{R}^{d}\right)} \leq C, \quad \forall t \in(0, T)
$$

for some constant $C$ not depending on $\Delta t$. To show compactness of the scheme, we must also show that $u_{\Delta t} \in C\left(0, T ; L^{1}\left(\mathbb{R}^{d}\right)\right)$ uniformly in $\Delta t$. In order to do this, we use the Kružkov interpolation lemma (Lem. 2.4). Let $\varphi(x)$ be a test function and set $\varphi_{I}=\varphi\left(x_{I}\right)$. Let $D_{t} X_{I}^{n}=X_{I}^{n+1}-X_{I}^{n}$ for any $X_{I}^{n}$. From (4.1) we find that

$$
\begin{aligned}
\sum_{I} D_{t} u_{I}^{n} \varphi_{I} h^{d} & =\sum_{I} \lambda \sum_{i=1}^{d} \widehat{\sum_{i}} f_{i}^{\mathrm{EO}}\left(k_{J-e_{i} / 2}, u_{I-e_{i}}^{n}, u_{I}^{n}\right) D_{i} \varphi_{I-e_{i}} h^{d}+\mu \sum_{i=1}^{d} D_{i} A\left(u_{I}^{n}\right) D_{i} \varphi_{I-e_{i}} h^{d} \\
& \leq C \Delta t\|\nabla \varphi\|_{L^{\infty}\left(\mathbb{R}^{d}\right)}\left(\sum_{I} \widehat{\sum_{i}} \sum_{i=1}^{d}\left|f_{i}^{\mathrm{EO}}\left(k_{J-e_{i} / 2}, u_{I-e_{i}}^{n}, u_{I}^{n}\right)\right| h^{d}+\sum_{I} \sum_{i=1}^{d}\left|D_{i} A\left(u_{I}^{n}\right)\right| h^{d-1}\right)
\end{aligned}
$$

In view of the uniform $L^{1}$ and $B V$ bounds in (4.3), an application of Lemma 2.4 gives

$$
\left\|u_{\Delta t}\left(\cdot, t_{1}\right)-u_{\Delta t}\left(\cdot, t_{2}\right)\right\|_{L^{1}\left(\mathbb{R}^{d}\right)} \leq C \sqrt{\left|t_{1}-t_{2}\right|}
$$

for some constant $C$ not depending on $\Delta t$. By Lemma 2.2, the sequence $\left\{u_{\Delta t}\right\}_{\Delta t>0}$ is compact in $L_{\text {loc }}^{1}\left(\Pi_{T}\right)$ and any limit point will satisfy (D.1) and (D.4).

We next show that the entropy condition (D.2) holds. To this end, a cell entropy inequality for the scheme (4.1) is established in the same way as in the hyperbolic case. A modification of the arguments leading to equation (3.15) yields

$$
\begin{aligned}
& \frac{U\left(u_{I}^{n+1}\right)-U\left(u_{I}^{n}\right)}{\Delta t}+\sum_{i=1}^{d} \widehat{\sum_{i}} \frac{\tilde{q}_{i}^{\mathrm{EO}}\left(k_{J+e_{i} / 2}^{i}, u_{I}^{n}, u_{I+e_{i}}^{n}\right)-\tilde{q}_{i}^{\mathrm{EO}}\left(k_{J-e_{i} / 2}^{i}, u_{I-e_{i}}^{n}, u_{I}^{n}\right)}{h} \\
& -\sum_{i=1}^{d} \frac{r\left(u_{I+e_{i}}^{n}\right)-2 r\left(u_{I}^{n}\right)+r\left(u_{I-e_{i}}^{n}\right)}{h^{2}}+U^{\prime}\left(u_{I}^{n+1}\right) \sum_{i=1}^{d} \widehat{\sum_{i}} \frac{f_{i}^{\mathrm{EO}}\left(k_{J+e_{i} / 2}^{i}, u_{I}^{n}, u_{I+e_{i}}^{n}\right)-f_{i}^{\mathrm{EO}}\left(k_{J-e_{i} / 2}^{i}, u_{I}^{n}, u_{I+e_{i}}^{n}\right)}{h} \\
& -\sum_{i=1}^{d} \widehat{\sum_{i}} \frac{\tilde{q}_{i}^{\mathrm{EO}}\left(k_{J+e_{i} / 2}^{i}, u_{I}^{n}, u_{I+e_{i}}^{n}\right)-\tilde{q}_{i}^{\mathrm{EO}}\left(k_{J-e_{i} / 2}^{i}, u_{I}^{n}, u_{I+e_{i}}^{n}\right)}{h} \leq 0, \quad(4.5)
\end{aligned}
$$


for any convex $C^{2}$ entropy $U$ and corresponding numerical entropy fluxes $\tilde{q}_{i}^{\mathrm{EO}}$ defined in (3.16) and $r$ defined by $r^{\prime}(u)=U^{\prime}(u) A^{\prime}(u)$. Consequently, any limit point of $\left\{u_{\Delta t}\right\}_{\Delta t>0}$ satisfies

$$
\iint_{\Pi_{T}}\left(U(u) \varphi_{t}+q(k, u) \cdot \nabla \varphi+r(u) \Delta \varphi-\left(U^{\prime}(u) f_{x}(k, u)-q_{x}(k, u)\right) \varphi\right) \mathrm{d} t \mathrm{~d} x \geq 0,
$$

where $q$ obeys $\partial_{u} q(k, u)=U^{\prime}(u) \partial_{u} f(k, u)$ and $f_{x}, q_{x}$ denote the divergence of $f(k(\cdot), u), q(k(\cdot), u)$, respectively. Since (4.6) holds for any convex $C^{2}$ entropy $U$ and corresponding entropy fluxes $q, r$, the usual approximation argument will show that (2.4) holds $\forall c \in \mathbb{R}$.

It remains to show that a limit $u$ of $\left\{u_{\Delta t}\right\}_{\Delta t>0}$ satisfies (D.3). This will be done by deriving a weak $B V$ estimate $[1,7,21,22]$. Multiplying (4.1) by $u_{I}^{n} h^{d}$, and summing over $I$, we find that

$$
\begin{array}{r}
\sum_{I}\left[u_{I}^{n} D_{t} u_{I}^{n} h^{d}+\Delta t \sum_{i=1}^{d} \widehat{\sum_{i} u_{I}^{n}\left(f_{i}^{\mathrm{EO}}\left(k_{J+e_{i} / 2}^{i}, u_{I}^{n}, u_{I+e_{i}}^{n}\right)-f_{i}^{\mathrm{EO}}\left(k_{J+e_{i} / 2}^{i}, u_{I-e_{i}}^{n}, u_{I}^{n}\right)\right) h^{d-1}}\right. \\
\left.+\mu \sum_{i=1}^{d} D_{i} A\left(u_{I-e_{i}}^{n}\right) D_{i} u_{I-e_{i}}^{n}\right]=-\Delta t \sum_{I}\left[\sum _ { i = 1 } ^ { d } \widehat { \sum _ { i } } u _ { I } ^ { n } \left(f_{i}^{\mathrm{EO}}\left(k_{J+e_{i} / 2}^{i}, u_{I-e_{i}}^{n}, u_{I}^{n}\right)\right.\right. \\
\left.\left.-f_{i}^{\mathrm{EO}}\left(k_{J-e_{i} / 2}^{i}, u_{I-e_{i}}^{n}, u_{I}^{n}\right)\right) h^{d-1}\right] .
\end{array}
$$

We can write

$$
u_{I}^{n} D_{t} u_{I}^{n}=\frac{1}{2}\left(D_{t}\left(u_{I}^{n}\right)^{2}-\left(D_{t} u_{I}^{n}\right)^{2}\right)
$$

and we also have that

$$
D_{i} A\left(u_{I-e_{i}}^{n}\right) D_{i} u_{I-e_{i}}^{n} \geq \frac{\left(D_{i} A\left(u_{I-e_{i}}^{n}\right)\right)^{2}}{\max _{u} A^{\prime}(u)}
$$

since $A^{\prime}(u) \geq 0$. Using these observations, we find that

$$
\begin{aligned}
\frac{\Delta t h^{d}}{\max _{u} A^{\prime}(u)} \sum_{I} \sum_{i=1}^{d}( & \left.\frac{D_{i} A\left(u_{I-e_{i}}^{n}\right)}{h}\right)^{2}+\Delta t \sum_{I} \sum_{i=1}^{d} \widehat{\sum}_{i} u_{I}^{n}\left(f_{i}^{\mathrm{EO}}\left(k_{J+e_{i} / 2}^{i}, u_{I}^{n}, u_{I+e_{i}}^{n}\right)\right. \\
& \left.-f_{i}^{\mathrm{EO}}\left(k_{J+e_{i} / 2}^{i}, u_{I-e_{i}}^{n}, u_{I}^{n}\right)\right) h^{d-1} \leq \frac{-h^{d}}{2} \sum_{I}\left[D_{t}\left(u_{I}^{n}\right)^{2}-\left(D_{t} u_{I}^{n}\right)^{2}\right] \\
& -\Delta t \sum_{I} \sum_{i=1}^{d} \widehat{\sum_{i}} u_{I}^{n}\left(f_{i}^{\mathrm{EO}}\left(k_{J+e_{i} / 2}^{i}, u_{I-e_{i}}^{n}, u_{I}^{n}\right)-f_{i}^{\mathrm{EO}}\left(k_{J-e_{i} / 2}^{i}, u_{I-e_{i}}^{n}, u_{I}^{n}\right)\right) h^{d-1} .
\end{aligned}
$$

Before we proceed, we note that

$$
f_{i}^{\mathrm{EO}}(k, v, w)-f_{i}^{\mathrm{EO}}(k, u, v)=\left(f_{i}^{-}(k, w)-f_{i}^{-}(k, v)\right)+\left(f_{i}^{+}(k, v)-f_{i}^{+}(k, u)\right),
$$

where

$$
f_{i}^{-}(k, u)=\int_{0}^{u}\left(\partial_{u} f_{i}(k, s) \wedge 0\right) \mathrm{d} s, \quad f_{i}^{+}(k, u)=\int_{0}^{u}\left(\partial_{u} f_{i}(k, s) \vee 0\right) \mathrm{d} s .
$$


Thus we can write inequality (4.7) as

$$
\begin{aligned}
\frac{\Delta t h^{d}}{\max _{u} A^{\prime}(u)} \sum_{I} \sum_{i=1}^{d}\left(\frac{D_{i} A\left(u_{I-e_{i}}^{n}\right)}{h}\right)^{2} & +\Delta t h^{d-1} \sum_{I} \sum_{i=1}^{d} \widehat{\sum_{i}}\left[u_{I}^{n}\left(f_{i}^{-}\left(k_{J+e_{i} / 2}^{i}, u_{I+e_{i}}^{n}\right)-f_{i}^{-}\left(k_{J+e_{i} / 2}^{i}, u_{I}^{n}\right)\right)\right. \\
& \left.+u_{I}^{n}\left(f_{i}^{+}\left(k_{J+e_{i} / 2}^{i}, u_{I}^{n}\right)-f_{i}^{+}\left(k_{J+e_{i} / 2}^{i}, u_{I-e_{i}}^{n}\right)\right)\right] \\
& \leq \frac{-h^{d}}{2} \sum_{I}\left[D_{t}\left(u_{I}^{n}\right)^{2}-\left(D_{t} u_{I}^{n}\right)^{2}\right]+C \Delta t \sum_{I} \sum_{i=1}^{d}\left|D_{i} k_{I-e_{i} / 2}^{i}\right| h^{d-1},(4.10
\end{aligned}
$$

for some constant $C$. The sum in (4.9) can be analyzed further by introducing the functions

$$
\mathcal{F}_{i}^{ \pm}(k, u)=\int_{0}^{u} s \partial_{u} f^{ \pm}(k, s) \mathrm{d} s
$$

Then an integration by parts reveals that

$$
\mathcal{F}_{i}^{ \pm}(k, b)-\mathcal{F}_{i}^{ \pm}(k, a)=b\left(f_{i}^{ \pm}(k, b)-f_{i}^{ \pm}(k, a)\right)-\int_{a}^{b}\left(f_{i}^{ \pm}(k, s)-f_{i}^{ \pm}(k, a)\right) \mathrm{d} s
$$

Therefore

$$
\begin{aligned}
u_{I}^{n}\left(f_{i}^{+}\left(k_{J+e_{i} / 2}^{i}, u_{I}^{n}\right)-f_{i}^{+}\left(k_{J+e_{i} / 2}^{i}, u_{I-e_{i}}^{n}\right)\right)= & \mathcal{F}_{i}^{+}\left(k_{J+e_{i} / 2}^{i}, u_{I}^{n}\right)-\mathcal{F}_{i}^{+}\left(k_{J+e_{i} / 2}^{i}, u_{I-e_{i}}^{n}\right) \\
& +\int_{u_{I-e_{i}}^{n}}^{u_{I}^{n}}\left(f_{i}^{+}\left(k_{J+e_{i} / 2}^{i}, s\right)-f_{i}^{+}\left(k_{J+e_{i} / 2}^{i}, u_{I-e_{i}}^{n}\right)\right) \mathrm{d} s \\
u_{I}^{n}\left(f_{i}^{-}\left(k_{J+e_{i} / 2}^{i}, u_{I+e_{i}}^{n}\right)-f_{i}^{-}\left(k_{J+e_{i} / 2}^{i}, u_{I}^{n}\right)\right)= & \mathcal{F}_{i}^{-}\left(k_{J+e_{i} / 2}^{i}, u_{I+e_{i}}^{n}\right)-\mathcal{F}_{i}^{-}\left(k_{J+e_{i} / 2}^{i}, u_{I}^{n}\right) \\
& -\int_{u_{I+e_{i}}^{n}}^{u_{I}^{n}}\left(f_{i}^{-}\left(k_{J+e_{i} / 2}^{i}, s\right)-f_{i}^{-}\left(k_{J+e_{i} / 2}^{i}, u_{I+e_{i}}^{n}\right)\right) \mathrm{d} s
\end{aligned}
$$

Consequently (4.9) can be written

$$
\begin{aligned}
\Delta t h^{d-1} \sum_{I} \sum_{i=1}^{d} \widehat{\sum_{i}}[ & \mathcal{F}_{i}^{+}\left(k_{J+e_{i} / 2}^{i}, u_{I}^{n}\right)-\mathcal{F}_{i}^{+}\left(k_{J+e_{i} / 2}^{i}, u_{I-e_{i}}^{n}\right)+\int_{u_{I-e_{i}}^{n}}^{u_{I}^{n}}\left(f_{i}^{+}\left(k_{J+e_{i} / 2}^{i}, s\right)\right. \\
& \left.-f_{i}^{+}\left(k_{J+e_{i} / 2}^{i}, u_{I-e_{i}}^{n}\right)\right) \mathrm{d} s+\mathcal{F}_{i}^{-}\left(k_{J+e_{i} / 2}^{i}, u_{I+e_{i}}^{n}\right)-\mathcal{F}_{i}^{-}\left(k_{J+e_{i} / 2}^{i}, u_{I}^{n}\right) \\
& \left.-\int_{u_{I+e_{i}}^{n}}^{u_{I}^{n}}\left(f_{i}^{-}\left(k_{J+e_{i} / 2}^{i}, s\right)-f_{i}^{-}\left(k_{J+e_{i} / 2}^{i}, u_{I+e_{i}}^{n}\right)\right) \mathrm{d} s\right] .
\end{aligned}
$$


We also have that $\mathcal{F}_{i}^{ \pm}$is locally Lipschitz continuous in $k$ as

$$
\left|\mathcal{F}_{i}^{ \pm}\left(k_{1}, u\right)-\mathcal{F}_{i}^{ \pm}\left(k_{2}, u\right)\right|=\left|\int_{0}^{u} s \partial_{u}\left(f_{i}^{ \pm}\left(k_{1}, s\right)-f_{i}^{ \pm}\left(k_{2}, s\right)\right) \mathrm{d} s\right| \leq \max _{k, u}\left|\partial_{u k} f_{i}\right||u|\left|k_{1}-k_{2}\right|
$$

Hence we obtain

$$
\begin{aligned}
\left|\sum_{I} \sum_{i=1}^{d} \widehat{\sum_{i}}\left[\mathcal{F}_{i}^{ \pm}\left(k_{J+e_{i} / 2}^{i}, u_{I}^{n}\right)-\mathcal{F}_{i}^{ \pm}\left(k_{J-e_{i} / 2}^{i}, u_{I}^{n}\right)\right] h^{d-1}\right| \\
\leq L_{u k} \max _{t}\left\|u_{\Delta t}(\cdot, t)\right\|_{L^{\infty}\left(\mathbb{R}^{d}\right)} \sum_{I} \sum_{i=1}^{d}\left|D_{i} k_{J-e_{i}}^{i}\right| h^{d-1},
\end{aligned}
$$

which is bounded uniformly in $\Delta t$. To bound the terms involving integrals, we need the following technical lemma (whose easy proof can be found in [22]):

Lemma 4.1. Let $h: \mathbb{R} \rightarrow \mathbb{R}$ be a monotone, Lipschitz continuous function, with a Lipschitz constant $L_{h}$. Then we have

$$
\left|\int_{a}^{b}(h(\xi)-h(a)) \mathrm{d} \xi\right| \geq \frac{1}{2 L_{h}}(h(b)-h(a))^{2}, \quad \forall a, b \in \mathbb{R} .
$$

Applying this to $f_{i}^{ \pm}$we find that

$$
\begin{aligned}
\int_{u_{I-e_{i}}^{n}}^{u_{I}^{n}}\left(f_{i}^{+}\left(k_{J+e_{i} / 2}^{i}, s\right)-\right. & \left.f_{i}^{+}\left(k_{J+e_{i} / 2}^{i}, u_{I-e_{i}}^{n}\right)\right) \mathrm{d} s \\
\geq & \frac{1}{2 \max _{u}\left|\partial_{u} f(k, u)\right|}\left(f_{i}^{+}\left(k_{J+e_{i} / 2}^{i}, u_{I}^{n}\right)-f_{i}^{+}\left(k_{J+e_{i} / 2}^{i}, u_{I-e_{i}}^{n}\right)\right)^{2}, \\
& -\int_{u_{I+e_{i}}^{n}}^{u_{I}^{n}}\left(f_{i}^{-}\left(k_{J+e_{i} / 2}^{i}, s\right)-f_{i}^{-}\left(k_{J+e_{i} / 2}^{i}, u_{I+e_{i}}^{n}\right)\right) \mathrm{d} s \\
\geq & \frac{1}{2 \max _{u}\left|\partial_{u} f(k, u)\right|}\left(f_{i}^{-}\left(k_{J+e_{i} / 2}^{i}, u_{I+e_{i}}^{n}\right)-f_{i}^{-}\left(k_{J+e_{i} / 2}^{i}, u_{I}^{n}\right)\right)^{2} .
\end{aligned}
$$

The above and (4.7) imply that

$$
\begin{array}{r}
\frac{\Delta t h^{d}}{\max _{u} A^{\prime}(u)} \sum_{I} \sum_{i=1}^{d}\left(\frac{D_{i} A\left(u_{I-e_{i}}^{n}\right)}{h}\right)^{2}+\frac{\Delta t h^{d-1}}{2 \max _{u, k}\left|\partial_{u} f(k, u)\right|} \sum_{I} \sum_{i=1}^{d} \widehat{\sum}_{i}\left[\left(f_{i}^{-}\left(k_{J+e_{i} / 2}^{i}, u_{I+e_{i}}^{n}\right)\right.\right. \\
\left.\left.-f_{i}^{-}\left(k_{J+e_{i} / 2}^{i}, u_{I}^{n}\right)\right)^{2}+\left(f_{i}^{+}\left(k_{J+e_{i} / 2}^{i}, u_{I}^{n}\right)-f_{i}^{+}\left(k_{J+e_{i} / 2}^{i}, u_{I-e_{i}}^{n}\right)\right)^{2}\right] \\
\leq \frac{-h^{d}}{2} \sum_{I}\left[D_{t}\left(u_{I}^{n}\right)^{2}-\left(D_{t} u_{I}^{n}\right)^{2}\right]+C \Delta t,
\end{array}
$$


where the constant $C$ does not depend on $\Delta t$. Furthermore, using the definition of the scheme, (4.1), and the inequality $(a+b)^{2} \leq 2\left(a^{2}+b^{2}\right)$, we find that

$$
\begin{aligned}
\frac{1}{2}\left(D_{t} u_{I}^{n}\right)^{2} \leq & 2 \lambda^{2} d \sum_{i=1}^{d} \widehat{\sum}_{i}\left[\left(f_{i}^{\mathrm{EO}}\left(k_{J+e_{i} / 2}^{i}, u_{I}^{n}, u_{I+e_{i}}^{n}\right)-f_{i}^{\mathrm{EO}}\left(k_{J+e_{i} / 2}^{i}, u_{I-e_{i}}^{n}, u_{I}^{n}\right)\right)^{2}\right. \\
& \left.+\left(f_{i}^{\mathrm{EO}}\left(k_{J+e_{i} / 2}^{i}, u_{I-e_{i}}^{n}, u_{I}^{n}\right)-f_{i}^{\mathrm{EO}}\left(k_{J-e_{i} / 2}^{i}, u_{I-e_{i}}^{n}, u_{I}^{n}\right)\right)^{2}\right] \\
& +2 \mu^{2} d \sum_{i=1}^{d}\left[\left(D_{i} A\left(u_{I}^{n}\right)\right)^{2}+\left(D_{i} A\left(u_{I-e_{i}}^{n}\right)\right)^{2}\right] \\
\leq & 4 \lambda^{2} d \sum_{i=1}^{d} \widehat{\sum_{i}}\left[\left(f_{i}^{-}\left(k_{J+e_{i} / 2}^{i}, u_{I+e_{i}}^{n}\right)-f_{i}^{-}\left(k_{J+e_{i} / 2}^{i}, u_{I}^{n}\right)\right)^{2}\right. \\
& \left.+\left(f_{i}^{+}\left(k_{J+e_{i} / 2}^{i}, u_{I}^{n}\right)-f_{i}^{+}\left(k_{J+e_{i} / 2}^{i}, u_{I-e_{i}}^{n}\right)\right)^{2}\right] \\
& +2 \lambda^{2} d \sum_{i=1}^{d} \widehat{\sum_{i}}\left(f_{i}^{\mathrm{EO}}\left(k_{J+e_{i} / 2}^{i}, u_{I-e_{i}}^{n}, u_{I}^{n}\right)-f_{i}^{\mathrm{EO}}\left(k_{J-e_{i} / 2}^{i}, u_{I-e_{i}}^{n}, u_{I}^{n}\right)\right)^{2} \\
& +2 \mu^{2} d \sum_{i=1}^{d}\left[\left(D_{i} A\left(u_{I}^{n}\right)\right)^{2}+\left(D_{i} A\left(u_{I-e_{i}}^{n}\right)\right)^{2}\right] .
\end{aligned}
$$

In what follows, we assume the following strengthened CFL condition

$$
\mathrm{CFL}_{\varepsilon}:=8 \lambda d \max _{i, k, u}\left|\partial_{u} f_{i}(k, u)\right|+4 \mu d \max _{u} A^{\prime}(u) \leq 1-\varepsilon,
$$

where $\varepsilon \in(0,1)$ is given a real number. Note that if $A^{\prime} \equiv 0$, i.e., in the hyperbolic case, (4.16) implies $\mathrm{CFL}_{\varepsilon} \in\left(0, \frac{1}{8}\right)$, which should be compared with the usual CFL $\in(0,1)$, see (4.2). The new CFL condition implies in particular that

$$
\begin{aligned}
& 4 \lambda^{2} d=4 \lambda d \frac{\Delta t}{h}=8 \lambda d \max _{k, u, i}\left|\partial_{u} f_{i}(k, u)\right| \frac{\Delta t}{2 h \max _{k, u, i}\left|\partial_{u} f_{i}(k, u)\right|} \leq \frac{\Delta t(1-\varepsilon)}{2 h \max _{k, u, i}\left|\partial_{u} f_{i}(k, u)\right|}, \\
& 2 \mu^{2} d=2 \mu d \frac{\Delta t}{h^{2}}=4 d \max _{u} A^{\prime}(u) \frac{\Delta t}{2 h^{2} \max _{u} A^{\prime}(u)} \leq \frac{\Delta t(1-\varepsilon)}{2 h^{2} \max _{u} A^{\prime}(u)},
\end{aligned}
$$

and therefore

$$
\begin{aligned}
\frac{1}{2}\left(D_{t} u_{I}^{n}\right)^{2} \leq & \frac{\Delta t(1-\varepsilon)}{2 h \max _{k, u, i}\left|\partial_{u} f_{i}(k, u)\right|} \sum_{i=1}^{d} \widehat{\sum}_{i}\left[\left(f_{i}^{+}\left(k_{J+e_{i} / 2}^{i}, u_{I}^{n}\right)-f_{i}^{+}\left(k_{J+e_{i} / 2}^{i}, u_{I-e_{i}}^{n}\right)\right)^{2}\right. \\
& \left.+\left(f_{i}^{-}\left(k_{J+e_{i} / 2}^{i}, u_{I+e_{i}}^{n}\right)-f_{i}^{-}\left(k_{J+e_{i} / 2}^{i}, u_{I}^{n}\right)\right)^{2}\right] \\
& +\frac{\Delta t(1-\varepsilon)}{2 h \max _{k, u, i}\left|\partial_{u} f_{i}(k, u)\right|} \sum_{i=1}^{d} \widehat{\sum}_{i}\left(\text { const. } D_{i} k_{J-e_{i} / 2}^{i}\right)^{2} \\
& +\frac{\Delta t(1-\varepsilon)}{2 h^{2} \max _{u} A^{\prime}(u)} \sum_{i=1}^{d}\left[\left(D_{i} A\left(u_{I}^{n}\right)\right)^{2}+\left(D_{i} A\left(u_{I-e_{i}}^{n}\right)\right)^{2}\right]
\end{aligned}
$$


Now we multiply the above inequality (4.17) by $h^{d}$ and sum over $I$ and $n=0, \ldots, N$, and sum (4.14) over $n$, and add the results to find that

$$
\begin{aligned}
\frac{\Delta t \varepsilon h^{d}}{\max _{u} A^{\prime}(u)} \sum_{n, I} \sum_{i=1}^{d}\left(\frac{D_{i} A\left(u_{I-e_{i}}^{n}\right)}{h}\right)^{2}+\frac{\Delta t h^{d-1} \varepsilon}{2 \max _{i, k, u}\left|\partial_{u} f_{i}(k, u)\right|} \sum_{n, I} \sum_{i=1}^{d} \widehat{\sum}_{i}\left[\left(f_{i}^{+}\left(k_{J+e_{i} / 2}^{i}, u_{I}^{n}\right)\right.\right. \\
\left.\left.-f_{i}^{+}\left(k_{J+e_{i} / 2}^{i}, u_{I-e_{i}}^{n}\right)\right)^{2}+\left(f_{i}^{-}\left(k_{J+e_{i} / 2}^{i}, u_{I+e_{i}}^{n}\right)-f_{i}^{-}\left(k_{J+e_{i} / 2}^{i}, u_{I}^{n}\right)\right)^{2}\right] \\
\leq \frac{h^{d}}{2} \sum_{I}\left(u_{I}^{0}\right)^{2}+C T \leq \frac{1}{2}\left\|u_{0}\right\|_{L^{\infty}\left(\mathbb{R}^{d}\right)}\left\|u_{0}\right\|_{L^{1}\left(\mathbb{R}^{d}\right)}+C T \leq C,
\end{aligned}
$$

for some constant $C$ not depending on $\Delta t$. We thus have the following bound

$$
\Delta t h^{d} \sum_{n, I} \sum_{i=1}^{d}\left(\frac{D_{i} A\left(u_{I}^{n}\right)}{h}\right)^{2} \leq C,
$$

for some constant $C$ not depending on $\Delta t$.

Next let $I(x)$ be the multi-index such that $x \in \chi_{I}$. Then we have that $I(x+y)-I(x)=: J=\left(J_{1}, \ldots, J_{d}\right)$, and $|J h| \leq|y|+h$. Set $K_{i}=I+\left(J_{1}, \ldots, J_{i-1}, 0, \ldots, 0\right)$. Using this notation we can write

$$
A\left(u_{\Delta t}(x+y, t)\right)-A\left(u_{\Delta t}(x, t)\right)=A\left(u_{I(x+y)}^{n}\right)-A\left(u_{I(x)}^{n}\right)=\sum_{i=1}^{d} \sum_{j=1}^{J_{i}} D_{i} A\left(u_{K_{i}-(j-1) e_{i}}^{n}\right) .
$$

By the Cauchy-Schwartz inequality

$$
\left(A\left(u_{\Delta t}(x+y, t)\right)-A\left(u_{\Delta t}(x, t)\right)\right)^{2} \leq|J| \sum_{i=1}^{d} \sum_{j=1}^{J_{i}}\left(D_{j} A\left(u_{K_{i}-(j-1) e_{j}}^{n}\right)\right)^{2},
$$

where $|J| \sum_{i=1}^{d}=\left|J_{i}\right|$.

Hence using (4.19),

$$
\begin{aligned}
\iint_{\Pi_{T}}\left(A\left(u_{\Delta t}(x+y, t)\right)-A\left(u_{\Delta t}(x, t)\right)\right)^{2} \mathrm{~d} t \mathrm{~d} x & \leq \Delta t h^{d} \sum_{n, I}\left(A\left(u_{I\left(x_{I}+y\right)}^{n}\right)-A\left(u_{I\left(x_{I}\right)}^{n}\right)\right)^{2} \\
& \leq \Delta t h^{d}|J| \sum_{n, I} \sum_{i=1}^{d} \sum_{j=1}^{J_{i}}\left(D_{i} A\left(u_{K_{i}-(j-1) e_{i}}^{n}\right)\right)^{2} \\
& \leq|J| \Delta t h^{d} \sum_{n, I} \sum_{i=1}^{d} \sum_{j=1}^{J_{i}} \sum_{\ell=1}^{d}\left(D_{\ell} A\left(u_{K_{i}-(j-1) e_{i}}^{n}\right)\right)^{2} \\
& \leq(|J|)^{2} d \Delta t h^{d} \sum_{n, I} \sum_{i=1}^{d}\left(D_{i} A\left(u_{I}^{n}\right)\right)^{2} \leq C(|J| h)^{2},
\end{aligned}
$$

where the constant $C$ does not depend on $y$ or $h$. Noting that $|J| h \leq C(|y|+h)$, we find that

$$
\left\|A\left(u_{\Delta t}(\cdot+y, \cdot)\right)-A\left(u_{\Delta t}(\cdot, \cdot)\right)\right\|_{L^{2}\left(\Pi_{T}\right)} \leq C(|y|+h) .
$$


Next, we will use the weak space estimate (4.19) and the difference scheme itself to show that $A\left(u_{\Delta t}\right)$ is also $L^{2}$ continuous in time. Let $n(t)$ denote the integer such that $t \in\left[t_{n(t)}, t_{n(t)+1}\right)$. Then we have

$$
\begin{aligned}
& \iint_{\Pi_{T-\tau}}\left(A\left(u_{\Delta t}(x, t+\tau)\right)-A\left(u_{\Delta t}(x, t)\right)\right)^{2} \mathrm{~d} t \mathrm{~d} x \\
& \quad \leq \max _{u} A^{\prime}(u) \iint_{\Pi_{T-\tau}}\left(A\left(u_{\Delta t}(x, t+\tau)\right)-A\left(u_{\Delta t}(x, t)\right)\right)\left(u_{\Delta t}(x, t+\tau)-u_{\Delta t}(x, t)\right) \mathrm{d} t \mathrm{~d} x \\
& \quad=\max _{u} A^{\prime}(u) \int_{0}^{T-\tau} h^{d} \sum_{I}\left(A\left(u_{I}^{n(t+\tau)}\right)-A\left(u_{I}^{n(t)}\right)\right)\left(u_{I}^{n(t+\tau)}-u_{I}^{n(t)}\right) \mathrm{d} t .
\end{aligned}
$$

We denote the above integrand by $B(t)$ and write

$$
B(t)=h^{d} \sum_{I}\left(A\left(u_{I}^{n(t+\tau)}\right)-A\left(u_{I}^{n(t)}\right)\right) \sum_{n=n(t)}^{n(t)+n(\tau)-1} D_{t} u_{I}^{n}
$$

Then each term in the sum over $n$ above equals

$$
\begin{aligned}
h^{d} \sum_{I}( & \left.A\left(u_{I}^{n(t+\tau)}\right)-A\left(u_{I}^{n(t)}\right)\right) D_{t} u_{I}^{n} \\
= & -\Delta t h^{d-1} \sum_{I} \sum_{i=1}^{d} \widehat{\sum_{i}}\left[( A ( u _ { I } ^ { n ( t + \tau ) } ) - A ( u _ { I } ^ { n ( t ) } ) ) \left\{f_{i}^{-}\left(k_{J+e_{i} / 2}^{i}, u_{I+e_{i}}^{n}\right)\right.\right. \\
& \left.-f_{i}^{-}\left(k_{J+e_{i} / 2}^{i}, u_{I}^{n}\right)+f_{i}^{+}\left(k_{J+e_{i} / 2}^{i}, u_{I}^{n}\right)-f_{i}^{+}\left(k_{J+e_{i} / 2}^{i}, u_{I-e_{i}}^{n}\right)\right\} \\
& +\left(A\left(u_{I}^{n(t+\tau)}\right)-A\left(u_{I}^{n(t)}\right)\right) \\
& \left.\times\left\{f_{i}^{\mathrm{EO}}\left(k_{J+e_{i} / 2}^{i}, u_{I-e_{i}}^{n}, u_{I}^{n}\right)-f_{i}^{\mathrm{EO}}\left(k_{J-e_{i} / 2}^{i}, u_{I-e_{i}}^{n}, u_{I}^{n}\right)\right\}\right] \\
& +\Delta t h^{d-2} \sum_{I}\left(A\left(u_{I}^{n(t+\tau)}\right)-A\left(u_{I}^{n(t)}\right)\right) \sum_{i=1}^{d} D_{i}^{2} A\left(u_{I-e_{i}}^{n}\right) .
\end{aligned}
$$

We can do a partial summation in (4.22) to find that

$$
\begin{aligned}
|(4.22)| & =\Delta t h^{d-1}\left|\sum_{i=1}^{d} \widehat{\sum_{i}} D_{i}\left(A\left(u_{I-e_{i}}^{n(t+\tau)}\right)-A\left(u_{I-e i}^{n(t)}\right)\right) f_{i}^{\mathrm{EO}}\left(k_{J+e_{i} / 2}^{i}, u_{I-e_{i}}^{n}, u_{I}^{n}\right)\right| \\
& \leq \frac{\Delta t h^{d}}{2} \sum_{i=1}^{d} \widehat{\sum_{i}} f_{i}^{\mathrm{EO}}\left(k_{J+e_{i} / 2}^{i}, u_{I-e_{i}}^{n}, u_{I}^{n}\right)\left(\left(\frac{D_{i} A\left(u_{I-e_{i}}^{n(t+\tau)}\right)}{h}\right)^{2}+\left(\frac{D_{i} A\left(u_{I-e_{i}}^{n(t)}\right)}{h}\right)\right.
\end{aligned}
$$


In (4.25), one should recall that we have uniform control over the $L^{2}\left(\Pi_{T}\right)$ norm of the discrete gradient of $A\left(u_{\Delta t}\right)$, see (4.19). Since $k$ is of bounded variation,

$$
|(4.23)| \leq \Delta t C|k|_{B V\left(\mathbb{R}^{d}\right)},
$$

where $C$ does not depend on $\Delta t$. Regarding (4.24) we have that

$$
\begin{aligned}
|(4.24)| & =\Delta t h^{d-2}\left|\sum_{I} \sum_{i=1}^{d}-D_{i} A\left(u_{I-e_{i}}^{n(t+\tau)}\right) D_{i} A\left(u_{I-e_{i}}^{n}\right)+D_{i} A\left(u_{I-e_{i}}^{n(t)}\right) A\left(u_{I-e_{i}}^{n}\right)\right| \\
& \leq \Delta t h^{d-2}\left[\sum_{I} \sum_{i=1}^{d} \frac{1}{2}\left(D_{i} A\left(u_{I-e_{i}}^{n(t+\tau)}\right)\right)^{2}+\frac{1}{2}\left(D_{i} A\left(u_{I-e_{i}}^{n(t)}\right)\right)^{2}+\left(D_{i} A\left(u_{I-e_{i}}^{n}\right)\right)^{2}\right] .
\end{aligned}
$$

We can write

$$
\int_{0}^{T-\tau} B(t) \mathrm{d} t \leq(T-\tau) C n(\tau) \Delta t+\int_{0}^{T-\tau}\left(\frac{1}{2} B_{1}(t)+\frac{1}{2} B_{2}(t)+B_{3}(t)\right) \mathrm{d} t
$$

where $C$ does not depend on $\Delta t$ and $B_{1}, B_{2}, B_{3}$ are defined via (4.21) and (4.27). Now

$$
\begin{aligned}
\int_{0}^{T-\tau} B_{1}(t) \mathrm{d} t & =\int_{0}^{T-\tau} \Delta t h^{d-2} \sum_{I} \sum_{i=1}^{d} \sum_{n=n(t)}^{n(t)+n(\tau)-1}\left(D_{i} A\left(u_{I}^{n(t+\tau)}\right)\right)^{2} \mathrm{~d} t \\
& =\sum_{m=0}^{N-n(\tau)} \Delta t h^{d-2} \sum_{I} \sum_{i=1}^{d} \sum_{n=n\left(t_{m}\right)}^{n\left(t_{m}\right)+n(\tau)-1}\left(D_{i} A\left(u_{I}^{n\left(t_{m}+\tau\right)}\right)\right)^{2} \Delta t \\
& \leq(n(\tau)+1) \Delta t \Delta t h^{d-2} \sum_{n} \sum_{I} \sum_{i=1}^{d}\left(D_{i} A\left(u_{I}^{n}\right)\right)^{2} \\
& \leq C(\tau+\Delta t) .
\end{aligned}
$$

Similarly

$$
\int_{0}^{T-\tau} B_{2}(t) \mathrm{d} t \leq C(\tau+\Delta t)
$$

Finally

$$
\begin{aligned}
\int_{0}^{T-\tau} B_{3}(t) \mathrm{d} t & \leq \sum_{m=0}^{N-n(\tau)} \Delta t h^{d-2} \sum_{n=m}^{m+n(\tau)-1} \sum_{I} \sum_{i=1}^{d}\left(D_{i} A\left(u_{I}^{n}\right)\right)^{2} \Delta t \\
& =\Delta t \sum_{k=0}^{n(\tau)-1} \Delta t h^{d-2} \sum_{n=0}^{N-n(\tau)} \sum_{I} \sum_{i=1}^{d}\left(D_{i} A\left(u_{I}^{n+k}\right)\right)^{2} \\
& \leq C(\tau+\Delta t),
\end{aligned}
$$

where $C$ does not depend on $\Delta t$. Using the bounds (4.28-4.30), we find that

$$
\left\|A\left(u_{\Delta t}(\cdot, \cdot+\tau)\right)-A\left(u_{\Delta t}(\cdot, \cdot)\right)\right\|_{L^{2}\left(\mathbb{R}^{d} \times(0, T-\tau)\right)} \leq C \sqrt{\Delta t+\tau} .
$$

In view Lemma 2.3, we conclude that

$$
A\left(u_{\Delta t}\right) \rightarrow \bar{A} \text { strongly in } L_{\mathrm{loc}}^{2}(\mathbb{R} \times(0, T)) \text { as } \Delta t \downarrow 0 \text { and } \bar{A} \in L^{2}\left(0, T ; H^{1}(\mathbb{R})\right) \text {. }
$$


Equipped with the strong convergence $u_{\Delta t} \rightarrow u$ a.e., we conclude immediately that $\bar{A}=A(u)$ and thus (D.3) holds.

We sum up our results in the following theorem:

Theorem 4.1. Assume that $A, f$, and $k$ satisfy (2.1, 2.2), and (2.3), respectively. Furthermore, assume that $u_{0}$ is a function in $L^{1}\left(\mathbb{R}^{d}\right) \cap L^{\infty}\left(\mathbb{R}^{d}\right) \cap B V\left(\mathbb{R}^{d}\right)$. If the CFL condition (4.16) holds, then the piecewise constant approximate solutions (3.5) generated by the Engquist-Osher scheme (4.1) converge to the unique entropy solution of (1.1).

In the special case without coefficients, i.e., $k=1$, we can use our techniques to prove existence of an entropy solution without necessarily having initial data in $B V\left(\mathbb{R}^{d}\right)$. This can be done as follows. Assuming that $u_{0} \in L^{\infty}\left(\mathbb{R}^{d}\right) \cap L^{1}\left(\mathbb{R}^{d}\right)$, we study the problem

$$
u_{t}+\operatorname{div} f(u)=\Delta A(u), \quad u(x, 0)=u_{0}(x),
$$

where $f$ and $A$ are as before. We obtain the two first bounds in (4.3) as before. Fixing $\varepsilon \in \mathbb{R}^{d}$, we have

$$
\left\|u_{\Delta t}(\cdot+\varepsilon, t)-u_{\Delta t}(\cdot, t)\right\|_{L^{1}\left(\mathbb{R}^{d}\right)} \leq\left\|u_{0}(\cdot+\varepsilon)-u_{0}(\cdot)\right\|_{L^{1}\left(\mathbb{R}^{d}\right)} \leq \nu\left(|\varepsilon| ; u_{0}\right)
$$

since any function in $L^{\infty}\left(\mathbb{R}^{d}\right) \cap L^{1}\left(\mathbb{R}^{d}\right)$ has some modulus of continuity and the scheme is now translation invariant. Then we use Kružkov's interpolation lemma (Lem. 2.4) to show that $u_{\Delta t}$ also has a modulus of continuity in time. Next, we use the $L^{1}$ compactness lemma (Lem. 2.2) to show that $\left\{u_{\Delta t}\right\}_{\Delta t>0}$ has a subsequence that converges strongly in $L^{1}$ to a function that satisfies (D.1), (D.4), and the entropy condition (D.3). To finally show that the limit satisfies (D.3), note that to obtain the crucial estimates (4.20) and (4.31) we did not use a $B V$ bound on $u_{\Delta t}$. Thus we have shown the following theorem:

Theorem 4.2. Assume that the function $u_{0}$ is in $L^{\infty}\left(\mathbb{R}^{d}\right) \cap L^{1}\left(\mathbb{R}^{d}\right)$. If the CFL condition (4.16) holds, then then the piecewise constant approximate solutions (3.5) generated by the Engquist-Osher scheme (4.1) converge to the unique entropy solution of (4.32).

\section{REFERENCES}

[1] M. Afif and B. Amaziane, Convergence of finite volume schemes for a degenerate convection-diffusion equation arising in two-phase flow in porous media. Preprint (1999).

[2] F. Bouchut, F.R. Guarguaglini and R. Natalini, Diffusive BGK approximations for nonlinear multidimensional parabolic equations. Indiana Univ. Math. J. 49 (2000) 723-749.

[3] R. Bürger, S. Evje and K.H. Karlsen, On strongly degenerate convection-diffusion problems modeling sedimentationconsolidation processes. J. Math. Anal. Appl. 247 (2000) 517-556.

[4] M.C. Bustos, F. Concha, R. Bürger and E.M. Tory, Sedimentation and thickening: Phenomenological foundation and mathematical theory. Kluwer Academic Publishers, Dordrecht (1999).

[5] J. Carrillo, Entropy solutions for nonlinear degenerate problems. Arch. Rational Mech. Anal. 147 (1999) 269-361.

[6] C. Chainais-Hillairet, Finite volume schemes for a nonlinear hyperbolic equation. Convergence towards the entropy solution and error estimate. RAIRO-Modél. Math. Anal. Numér. 33 (1999) 129-156.

[7] S. Champier, T. Gallouët and R. Herbin, Convergence of an upstream finite volume scheme for a nonlinear hyperbolic equation on a triangular mesh. Numer. Math. 66 (1993) 139-157.

[8] B. Cockburn, F. Coquel and P. Le Floch, An error estimate for finite volume methods for multidimensional conservation laws. Math. Comp. 63 (1994) 77-103.

[9] B. Cockburn, F. Coquel and P.G. LeFloch, Convergence of the finite volume method for multidimensional conservation laws. SIAM J. Numer. Anal. 32 (1995) 687-705.

[10] B. Cockburn and P.-A. Gremaud, A priori error estimates for numerical methods for scalar conservation laws. I. The general approach. Math. Comp. 65 (1996) 533-573. 
[11] B. Cockburn and C.-W. Shu, The local discontinuous Galerkin method for time-dependent convection-diffusion systems. SIAM J. Numer. Anal. 35 (1998) 2440-2463 (electronic).

[12] M.G. Crandall and A. Majda, Monotone difference approximations for scalar conservation laws. Math. Comp. 34 (1980) 1-21.

[13] M.G. Crandall and L. Tartar, Some relations between nonexpansive and order preserving mappings. Proc. Amer. Math. Soc. 78 (1980) 385-390.

[14] B. Engquist and S. Osher, One-sided difference approximations for nonlinear conservation laws. Math. Comp. 36 (1981) 321-351.

[15] M.S. Espedal and K.H. Karlsen, Numerical solution of reservoir flow models based on large time step operator splitting algorithms, in Filtration in Porous media and industrial applications. Lect. Notes Math. 1734, Springer, Berlin (2000) 9-77.

[16] S. Evje and K.H. Karlsen, Discrete approximations of $B V$ solutions to doubly nonlinear degenerate parabolic equations. Numer. Math. 86 (2000) 377-417.

[17] S. Evje and K.H. Karlsen, Degenerate convection-diffusion equations and implicit monotone difference schemes, in Hyperbolic problems: Theory, numerics, applications, Vol. I (Zürich, 1998). Birkhäuser, Basel (1999) 285-294.

[18] S. Evje and K.H. Karlsen, Viscous splitting approximation of mixed hyperbolic-parabolic convection-diffusion equations. Numer. Math. 83 (1999) 107-137.

[19] S. Evje and K.H. Karlsen, Monotone difference approximations of $B V$ solutions to degenerate convection-diffusion equations. SIAM J. Numer. Anal. 37 (2000) 1838-1860 (electronic).

[20] S. Evje and K.H. Karlsen, Second order difference schemes for degenerate convection-diffusion equations. Preprint (in preparation).

[21] R. Eymard, T. Gallouët, M. Ghilani and R. Herbin, Error estimates for the approximate solutions of a nonlinear hyperbolic equation given by finite volume schemes. IMA J. Numer. Anal. 18 (1998) 563-594.

[22] R. Eymard, T. Gallouët, D. Hilhorst and Y. Naït Slimane, Finite volumes and nonlinear diffusion equations. RAIRO-Modél. Math. Anal. Numér. 32 (1998) 747-761.

[23] T. Gimse and N.H. Risebro, Solution of the Cauchy problem for a conservation law with a discontinuous flux function. SIAM J. Math. Anal. 23 (1992) 635-648.

[24] A. Harten, J.M. Hyman and P.D. Lax, On finite-difference approximations and entropy conditions for shocks. Comm. Pure Appl. Math. XXIX (1976) 297-322.

[25] H. Holden, K.H. Karlsen and K.-A. Lie, Operator splitting methods for degenerate convection-diffusion equations I: Convergence and entropy estimates, in Stochastic processes, physics and geometry: New interplays. A volume in honor of Sergio Albeverio. Amer. Math. Soc. (to appear).

[26] H. Holden, K.H. Karlsen, K.-A. Lie and N.H. Risebro, Operator splitting for nonlinear partial differential equations: An $L^{1}$ convergence theory. Preprint (in preparation).

[27] E. Isaacson and B. Temple, Convergence of the $2 \times 2$ Godunov method for a general resonant nonlinear balance law. SIAM J. Appl. Math. 55 (1995) 625-640.

[28] K.H. Karlsen and N.H. Risebro, On the uniqueness and stability of entropy solutions of nonlinear degenerate parabolic equations with rough coefficients. Preprint, Department of Mathematics, University of Bergen (2000).

[29] C. Klingenberg and N.H. Risebro, Stability of a resonant system of conservation laws modeling polymer flow with gravitation. J. Differential Equations March (2000).

[30] C. Klingenberg and N.H. Risebro, Convex conservation laws with discontinuous coefficients. Existence, uniqueness and asymptotic behavior. Comm. Partial Differential Equations 20 (1995) 1959-1990.

[31] D. Kröner, S. Noelle and M. Rokyta, Convergence of higher order upwind finite volume schemes on unstructured grids for scalar conservation laws in several space dimensions. Numer. Math. 71 (1995) 527-560.

[32] D. Kröner and M. Rokyta, Convergence of upwind finite volume schemes for scalar conservation laws in two dimensions. SIAM J. Numer. Anal. 31 (1994) 324-343.

[33] S.N. Kružkov, Results on the nature of the continuity of solutions of parabolic equations, and certain applications thereof. Mat. Zametki 6 (1969) 97-108.

[34] S.N. Kružkov, First order quasi-linear equations in several independent variables. Math. USSR Sbornik 10 (1970) $217-243$.

[35] A. Kurganov and E. Tadmor, New high-resolution central schemes for nonlinear conservation laws and convection-diffusion equations. J. Comput. Phys. 160 (2000) 241-282.

[36] N.N. Kuznetsov, Accuracy of some approximative methods for computing the weak solutions of a first-order quasi-linear equation. USSR Comput. Math. Math. Phys. Dokl. 16 (1976) 105-119.

[37] B.J. Lucier, Error bounds for the methods of Glimm, Godunov and LeVeque. SIAM J. Numer. Anal. 22 (1985) $1074-1081$.

[38] S. Noelle, Convergence of higher order finite volume schemes on irregular grids. Adv. Comput. Math. 3 (1995) $197-218$.

[39] M. Ohlberger, A posteriori error estimates for vertex centered finite volume approximations of convection-diffusion-reaction equations. Preprint, Mathematische Fakultät, Albert-Ludwigs-Universität Freiburg (2000).

[40] O.A. Oleŭnik, Discontinuous solutions of non-linear differential equations. Amer. Math. Soc Transl. Ser. 226 (1963) $95-172$.

[41] S. Osher and E. Tadmor, On the convergence of difference approximations to scalar conservation laws. Math. Comp. 50 (1988) $19-51$. 
[42] É. Rouvre and G. Gagneux, Solution forte entropique de lois scalaires hyperboliques-paraboliques dégénérées. $C$. $R$. Acad. Sci. Paris Sér. I Math. 329 (1999) 599-602.

[43] A.A. Samarskii, V.A. Galaktionov, S.P. Kurdyumov and A.P. Mikhailov, Blow-up in quasilinear parabolic equations. Walter de Gruyter \& Co., Berlin (1995). Translated from the 1987 Russian original by Michael Grinfeld and revised by the authors.

[44] R. Sanders, On convergence of monotone finite difference schemes with variable spatial differencing. Math. Comp. 40 (1983) 91-106.

[45] B. Temple, Global solution of the Cauchy problem for a class of $2 \times 2$ nonstrictly hyperbolic conservation laws. Adv. in Appl. Math. 3 (1982) 335-375.

[46] J. Towers, Convergence of a difference scheme for conservation laws with a discontinuous flux. Preprint, Available at the URL http://www.math.ntnu.no/conservation/

[47] J. Towers, A difference scheme for conservation laws with a discontinuous flux - the nonconvex case. Preprint, Available at the URL http://www.math.ntnu.no/conservation/

[48] J.-P. Vila, Convergence and error estimates in finite volume schemes for general multidimensional scalar conservation laws. I. Explicit monotone schemes. RAIRO-Modél. Math. Anal. Numér. 28 (1994) 267-295.

[49] A.I. Vol'pert, The spaces BV and quasi-linear equations. Math. USSR Sbornik 2 (1967) 225-267.

[50] A.I. Vol'pert and S.I. Hudjaev, Cauchy's problem for degenerate second order quasilinear parabolic equations. Math. USSR Sbornik 7 (1969) 365-387.

To access this journal online: www.edpsciences.org 